Peter Gerlinger, Lagrangian transported MDF methods for compressible high speed flows, Journal of computational Physics 339 (2017) 68-95.

The original publication is available at www.elsevier.com

http://www.sciencedirect.com/science/article/pii/S0021999117301523

http://dx.doi.org/10.1016/j.jcp.2017.02.049 


\title{
Lagrangian Transported MDF Methods for Compressible High Speed Flows
}

\author{
Peter Gerlinger* \\ Institute of Combustion Technology of Aerospace Engineering, University of Stuttgart, \\ Pfaffenwaldring 38-40, 70569 Stuttgart, Germany
}

\begin{abstract}
This paper deals with the application of thermochemical Lagrangian MDF (mass density function) methods for compressible sub- and supersonic RANS (Reynolds Averaged Navier-Stokes) simulations. A new approach to treat molecular transport is presented. This technique on the one hand ensures numerical stability of the particle solver in laminar regions of the flow field (e.g. in the viscous sublayer) and on the other hand takes differential diffusion into account. It is shown in a detailed analysis, that the new method correctly predicts first and second-order moments on the basis of conventional modeling approaches. Moreover, a number of challenges for MDF particle methods in high speed flows is discussed, e.g. high cell aspect ratio grids close to solid walls, wall heat transfer, shock resolution, and problems from statistical noise which may cause artificial shock systems in supersonic flows. A Mach 2 supersonic mixing channel with multiple shock reflection and a model rocket combustor simulation demonstrate the eligibility of this technique to practical applications. Both test cases are simulated successfully for the first time with a hybrid finite-volume
\end{abstract} (FV)/Lagrangian particle solver (PS).

Keywords: Mass density function (MDF), probability density function (PDF), high speed flow, compressible flow, combustion, rocket combustor

\footnotetext{
*Institute of Combustion Technology of Aerospace Engineering, University of Stuttgart, Pfaffenwaldring 38-40, 70569 Stuttgart, Germany

Email address: peter.gerlinger@dlr.de (Peter Gerlinger )
} 


\section{Introduction}

Since the pioneering work of Pope [1, 2], Lagrangian transported PDF (probability density function) or MDF (the MDF is a density weighted PDF) simulations are in use for more than three decades now. While in the beginning hybrid RANS/PDF simulations have been performed [3, 4, 5, 6, 7], the present trend is towards more complex and still more demanding LES (large-eddy simulation)/FMDF (filtered MDF) couplings [8, 9, 10,11]. Despite the large number of papers published on MDF methods, the greatest part of them investigate academic laboratory flames and/or simple geometric configurations only. Publications dealing with wall bounded flows, wall heat transfer, or even real combustors are rare. Some exceptions are [12, 11], [13], and [10], where internal combustion engines, gas turbine combustors, and swirling flows are simulated with MDF methods, respectively. Moreover, most papers investigate incompressible flows, where the pressure may be treated as thermodynamically constant. On the other hand there is a large demand for this technique to be used in compressible turbulent applications too, e.g. in rocket and supersonic combustion.

For reactive flows the most simple MDF approach is to describe the thermochemical variables (energy and gas composition) by a MDF, while solving conventionally averaged transport equations for the remaining variables needed. Such a hybrid approach is employed in the present paper, where Lagrangian particles represent the MDF.

The main cause why MDF methods are seldom used for compressible flows is the necessity to include the density (or pressure) into the set of independent random variables, or, to use simplifications. In the first case a conditional expectation of divergence appears in the MDF equation which is important but difficult to model. Eifler and Kollmann [14] presented a sophisticated technique which included density and dilatation as independent random variables in an Eularian PDF framework. However, this approach has not been employed afterwards in any practical application. Delarue and Pope $[15,16]$ used the pressure as a Lagrangian MDF variable, but again, this technique has hardly been used 
afterwards. One problem with their approach is the high degree of modeling required for the pressure equation. Another method is to neglect pressure fluctuations at the beginning of the MDF step. In incompressible low speed flows this is the usual procedure because in such cases pressure can be treated as constant in the MDF part. In compressible flows, however, one particle property (density or pressure) is missing, if the MDF covers the thermochemical variables energy and gas composition only. The reason is that the continuity equation, which is solved by the FV scheme, delivers the mean density only. Because of the missing information the particle pressures are initialized with the mean pressure of the volume obtained after the FV step by many authors $[5,17,18,19,20,21]$. Errors resulting from this simplification in high speed compressible flows are still unknown. This technique is chosen in the present paper and details will be given later. In the LES/FMDF technique of Banaeizadeh et al. $[22,11]$ the energy equation is solved with both the FV and the particle solver. To this end averaged source terms are transferred to the FV scheme. This is in contrast to the present approach, where the energy equation is solved by the particle method only. Still more demanding methods for compressible flows like the EPVS (energy-pressure-velocity-scalar) FMDF method [23] are under development.

Due to the much stronger coupling (caused by changes in pressure) between the MDF and the FV solver in compressible flows, there is also a greater impact of statistical noise from transferred averaged particle data to the FV scheme. This may cause stability problems. Moreover, some additional terms have to be modeled in compressible flows (i.e. work by viscous forces, pressure derivatives, and dissipation of turbulent kinetic energy), what is difficult to accomplish on an individual particle level. Instead, the corresponding terms are usually calculated from averaged FV data $[5,17,18,19]$. These terms are transferred to all particles of a volume in an identical way.

There are some additional challenges for MDF methods in supersonic flows: How to deal with shock waves and how to deal with highly stretched grids? Cell aspect ratios of up to 10,000 often occur in RANS simulations of super- 
and hypersonic flows close to solid walls. Because pure LES of high Reynolds number flows are extremely expensive, hybrid RANS/LES methods for the fluid flow, as e.g. the DDES (delayed detached-eddy simulation) [24], seem to be a feasible compromise for the foreseeable future. If coupled with a MDF scheme, the particle solver again has to deal with very high cell aspect ratios close to solid walls. A further point is shock capturing. Over the last 40 years sophisticated discretization techniques have been developed to achieve a sharp shock resolution with low numerical diffusion. Flux vector and flux difference splitting schemes achieve excellent results even if the shock is oblique with respect to the computational grid. WENO schemes [25] are able to reach high discretization orders directly at the shock. These techniques are not available on a particle level required for MDF scalar-velocity simulations.

Another problem is a correct prediction of laminar or weakly turbulent flows with MDF methods, which appear in case of relaminarization, close to solid walls, or in flows with a laminar surrounding. In such regions differential diffusion may become important. While in this paper RANS simulations are presented only, molecular transport and differential diffusion are essential in LES, too, where the differences between turbulent and molecular gas properties are much smaller than in RANS simulations.

This paper deals with most of the problems introduced above. For simplicity, a thermochemical MDF is used which covers energy and species mass fractions, while continuity, momentum, and turbulence equations are solved by a high order FV scheme. Because the focus is on numerical aspects of MDF methods and on a new approach for differential diffusion, predominantly non-reactive flows are investigated.

\section{Finite-volume scheme}

In the hybrid finite-volume/Lagrangian particle approach the in-house code TASCOM3D (Turbulent All Speed Combustion Multigrid solver) [26, 27, 28, 29] 
is used to solve the averaged continuity and momentum equations

$$
\begin{gathered}
\frac{\partial \bar{\rho}}{\partial t}+\frac{\partial}{\partial x_{i}}\left(\bar{\rho} \tilde{u}_{i}\right)=0 \\
\frac{\partial}{\partial t}\left(\bar{\rho} \tilde{u}_{i}\right)+\frac{\partial}{\partial x_{j}}\left(\bar{\rho} \tilde{u}_{i} \tilde{u}_{j}\right)=-\frac{\partial}{\partial x_{j}}\left(\bar{\rho} \widetilde{u_{i}^{\prime \prime} u_{j}^{\prime \prime}}\right)+\frac{\partial \bar{\tau}_{i j}}{\partial x_{j}}-\frac{\partial \bar{p}}{\partial x_{i}}
\end{gathered}
$$

as well as two transport equations of a turbulence model (in the present case the low-Reynolds number $q$ - $\omega$ model from Coakley and Huang [30] with $q=\sqrt{k}, k$ is the turbulent kinetic energy, and $\omega$ the specific dissipation rate). While the code allows both steady-state and time-accurate simulations, only steady-state results are presented in this paper. In the above equations $\rho$ is the density, $p$ the pressure, $u_{i}$ the velocity component in $i$-coordinate direction, and $\tau_{i j}$ is an element of the stress tensor. Further, $t$ is the time, $x_{i}$ are the coordinate directions, and ${ }^{-}$indicates Reynolds and ${ }^{\sim}$ Favre averages, respectively. In this paper Einstein summation is taken over the indices $i, j, k$, and $l$, but not over Greek indices. Moreover, $i$ and $j$ are exclusively used for the coordinate directions. An implicit LU-SGS (Lower-Upper Symmetric Gauss-Seidel) [31, 32, 33] scheme solves the set of equations, given above, in a coupled way. The discretization is up to third order in time by using a BDF (backward differentiation formula) technique. For spatial discretization a newly developed high order MLP (multi-dimensional limiting process) [34, 29] scheme is employed, which is up to sixth order in space and offers high accuracy and robustness while keeping the computational cost low. The fluxes at cell interfaces are calculated using the $\mathrm{AUSM}^{+}$-up flux vector splitting of Liou [35]. TASCOM3D works with structured grids. The code is parallelized using MPI (Message Passing Interface) and shows a good performance on both vector processors and massively parallel scalar architectures.

The different steps of the FV solver and the data obtained from and transferred to the particle solver (PS) are shown on the left side of Fig. 1. In the FV part of the hybrid scheme $\bar{\rho}$ and $\tilde{u}_{i}$ are advanced, while gas composition, enthalpy, and temperature are frozen. From the new density, frozen gas composition, and frozen temperature a new pressure is calculated from the equation 


\begin{tabular}{|c|c|c|c|}
\hline \multicolumn{2}{|c|}{ FV solver } & \multicolumn{2}{|c|}{ Particle solver (PS) } \\
\hline $\bar{\rho}^{n}, \widetilde{u}_{i}^{n}, q^{n}, \omega^{n}$ & $\begin{array}{l}\text { from last } \\
\text { FV step }\end{array}$ & $h^{p, n}, Y_{\alpha}^{p, n}, T^{p, n}$ & $\begin{array}{l}\text { from last } \\
\text { PS step }\end{array}$ \\
\hline$\widetilde{T}^{n}, \Theta_{T Y}^{n}, \bar{p}^{n}, \mu^{n}$ & $\begin{array}{l}\text { from last } \\
\text { PS step }\end{array}$ & $\bar{\rho}^{n+1}, \widetilde{u}_{i}^{n+1}, \tau^{n+1}, \bar{p}^{1}$ & $\begin{array}{c}\text { from FV } \\
\text { solver }\end{array}$ \\
\hline \multicolumn{2}{|c|}{$\begin{array}{l}\text { Implicit solver: continuity, } \\
\text { momentum, turbulence Eqs. } \\
\Rightarrow \bar{\rho}^{n+1}, \widetilde{u}_{i}^{n+1}, q^{n+1}, \omega^{n+1} \\
\Rightarrow \tau^{n+1}=1 / \omega^{n+1} \\
\text { EOS: } \bar{p}^{1}=p\left(\bar{\rho}^{n+1}, \widetilde{T}^{n}, \Theta_{T Y}^{n}\right)\end{array}$} & \multirow{6}{*}{\multicolumn{2}{|c|}{$\begin{array}{l}\text { Assumption: } p^{p, 1}=\bar{p}^{1} \\
\text { for all particles } p=1,2, \ldots, M \\
\text { EOS: } \rho^{p, 1}=\rho\left(Y_{\alpha}^{p, n}, T^{p, n}, p^{p, 1}\right) \\
\text { FS Ch: } \Rightarrow Y_{\alpha}^{p, 1}, h^{p, 1}=h^{p, n} \\
\text { FS M: } \Rightarrow Y_{\alpha}^{p, 2}, h^{p, 2} \\
\text { FS Co: } \Rightarrow Y_{\alpha}^{p, n+1}, h^{p, n+1}, T^{p, n+1} \\
\text { FS T: } \Rightarrow x_{i}^{p, n+1} \\
\text { Averaging: (IA and MTA) } \\
\Rightarrow \widetilde{Y}_{\alpha}^{n+1}, \widetilde{T}^{n+1}, \Theta_{T Y}^{n+1} \\
\text { Implicit smoothing }(\text { optional): } \\
\Rightarrow \widetilde{Y}_{\alpha}^{n+1}, \widetilde{T}^{n+1}, \Theta_{T Y}^{n+1} \\
\text { EOS: } \bar{p}^{n+1}=p\left(\bar{\rho}^{n+1}, \widetilde{T}^{n+1}, \Theta_{T Y}^{n+1}\right) \\
\text { Gas properties: } \mu^{n+1}, \lambda^{n+1}, D_{\alpha}^{n+1}\end{array}$}} \\
\hline \multirow[t]{6}{*}{$\bar{\rho}^{n+1}, \widetilde{u}_{i}^{n+1}, \tau^{n+1}$} & \multirow[t]{5}{*}{ to PS } & & \\
\hline & & & \\
\hline & & & \\
\hline & & & \\
\hline & & & \\
\hline & & $\widetilde{T}^{n+1}, \Theta_{T Y}^{n+1}, \bar{p}^{n+1}, \mu^{n+}$ & $\begin{array}{l}\text { to FV } \\
\text { solver }\end{array}$ \\
\hline
\end{tabular}

Figure 1: Diagram of the sequence of one iteration of the hybrid scheme for a steady-state simulation (EOS - equation of state, FS - fractional step, $C h$ - chemistry, $M$ - turbulent mixing, Co - changes due to compressibility, viscous dissipation, and differential diffusion, $T$ - spatial transport, $I A$ - instantaneous averaging, $M T A$ - moving-time averaging).

of state (EOS)

$$
\bar{p}=\overline{\rho R_{m} T \sum_{\alpha=1}^{N} \frac{Y_{\alpha}}{M_{\alpha}}}=\bar{\rho} R_{m}\left(\widetilde{T} \sum_{\alpha=1}^{N} \frac{\widetilde{Y}_{\alpha}}{M_{\alpha}}+\sum_{\alpha=1}^{N} \frac{1}{M_{\alpha}} \widetilde{T^{\prime \prime} Y_{\alpha}^{\prime \prime}}\right),
$$

taking species temperature correlations into account. In this equation $T$ denotes temperature, $Y_{\alpha}$ is the mass fraction of species $\alpha(\alpha=1,2, \ldots, N$, where $N$ is the number of species), $M_{\alpha}$ the corresponding molecular weight, and $R_{m}$ the universal gas constant. The temperature-species correlation term (the last sum on the right-hand side of Eq. (3)) is usually neglected in RANS simulations. In 
the framework of the hybid FV/Lagrangian particle solver Eq. (3) is replaced by

$$
\bar{p}=\bar{\rho} R_{m} \Theta_{T Y}
$$

with

$$
\Theta_{T Y}=\frac{1}{\sum_{p=1}^{M} m^{p}} \sum_{p=1}^{M}\left(m^{p} T^{p} \sum_{\alpha=1}^{N} \frac{Y_{\alpha}^{p}}{M_{\alpha}}\right),
$$

where $m^{p}$ are individual particle masses and $M$ is the total number of particles in the corresponding volume. $\Theta_{T Y}$ is calculated after the MDF step and is kept constant during the FV simulation. After a new pressure is calculated according to Eq. (4), the new mean pressure together with the updated values of mean density, velocity, and a turbulence time scale $\tau$ (obtained from the specific dissipation rate $\omega$ ) are transferred to the MDF solver (see Fig. 1). The computational grid defines the volumes for the FV solver (here also referred to as cells). Based on these volumes Ensemble averages or other mean values are calculated in the particle solver using all particles with are located inside the cell.

\section{First and second moment equations}

In the present approach the scalar MDF is based on the thermochemical variables enthalpy and species mass fractions. The corresponding balance equations of these variables are given by

$$
\begin{aligned}
\frac{\partial}{\partial t}(\rho h)+\frac{\partial}{\partial x_{i}}\left(\rho u_{i} h\right) & =\frac{\partial p}{\partial t}+u_{i} \frac{\partial p}{\partial x_{i}}+\tau_{i j} \frac{\partial u_{i}}{\partial x_{j}}-\frac{\partial q_{i}}{\partial x_{i}} \\
\frac{\partial}{\partial t}\left(\rho Y_{\alpha}\right)+\frac{\partial}{\partial x_{i}}\left(\rho u_{i} Y_{\alpha}\right) & =-\frac{\partial j_{\alpha i}}{\partial x_{i}}+S_{\alpha} .
\end{aligned}
$$

Here $h$ is the enthalpy, $S_{\alpha}$ the chemical source term, $q_{i}$ is the heat flux in $i$-direction which is calculated by

$$
q_{i}=-\lambda \frac{\partial T}{\partial x_{i}}+\sum_{\alpha=1}^{N} h_{\alpha} j_{\alpha i}
$$


and the diffusive flux of species $\alpha$ in $i$-direction is obtained from

$$
j_{\alpha i}=-\rho D_{\alpha} \frac{\partial Y_{\alpha}}{\partial x_{i}}+Y_{\alpha} \sum_{\beta=1}^{N} \rho D_{\beta} \frac{\partial Y_{\beta}}{\partial x_{i}} .
$$

The sum in Eq. (9) is added in order to achieve $\sum_{\alpha} j_{\alpha i}=0$ [36]. This term adds a correction which is weighted with the mass fraction of the corresponding species.

For a later evaluation of modeling approaches for unclosed terms in the MDF equation, transport equations for the first and second moments of the thermochemical variables are needed for comparison. Starting from Eqs. (6) and (7) exact but unclosed equations for the Favre averaged enthalpy $\widetilde{h}$ and species mass fractions $\tilde{Y}_{\alpha}$ are derived [37]

$$
\begin{aligned}
\frac{\partial}{\partial t}(\bar{\rho} \widetilde{h})+\frac{\partial}{\partial x_{i}}\left(\bar{\rho} \widetilde{u}_{i} \widetilde{h}\right)= & \frac{\partial \bar{p}}{\partial t}+\widetilde{u}_{i} \frac{\partial \bar{p}}{\partial x_{i}}+\overline{u_{i}^{\prime \prime} \frac{\partial p}{\partial x_{i}}}+\bar{\tau}_{i j} \frac{\partial \widetilde{u}_{i}}{\partial x_{j}}+\overline{\tau_{i j} \frac{\partial u_{i}^{\prime \prime}}{\partial x_{j}}} \\
& -\frac{\partial}{\partial x_{i}}\left(\bar{q}_{i}+\bar{\rho} \widetilde{u_{i}^{\prime \prime} h^{\prime \prime}}\right) \\
\frac{\partial}{\partial t}\left(\bar{\rho} \widetilde{Y}_{\alpha}\right)+\frac{\partial}{\partial x_{i}}\left(\bar{\rho} \widetilde{u}_{i} \widetilde{Y}_{\alpha}\right)= & -\frac{\partial}{\partial x_{i}}\left(\bar{j}_{\alpha i}+\widetilde{\rho} \widetilde{u_{i}^{\prime \prime} Y_{\alpha}^{\prime \prime}}\right)+\bar{S}_{\alpha}
\end{aligned}
$$

The averaged molecular heat and mass fluxes are usually approximated by

$$
\begin{aligned}
\bar{q}_{i} & \approx-\lambda \frac{\partial \tilde{T}}{\partial x_{i}}+\sum_{\alpha=1}^{N} \tilde{h}_{\alpha} \bar{j}_{\alpha i}, \\
\bar{j}_{\alpha i} & \approx-\bar{\rho} D_{\alpha} \frac{\partial \tilde{Y}_{\alpha}}{\partial x_{i}}+\tilde{Y}_{\alpha} \sum_{\beta=1}^{N} \bar{\rho} D_{\beta} \frac{\partial \tilde{Y}_{\beta}}{\partial x_{i}},
\end{aligned}
$$

where the influence of turbulence on transport properties is neglected. Instead the heat conductivity $\lambda$ and the diffusion coefficients $D_{\alpha}$ are calculated from averaged values.

In addition, second-order moments are needed. In the case of enthalpy the transport equation of enthalpy variance $\sigma_{h}=\widetilde{h^{\prime \prime}}$ becomes

$$
\frac{\partial}{\partial t}\left(\bar{\rho} \sigma_{h}\right)+\frac{\partial}{\partial x_{i}}\left(\bar{\rho} \widetilde{u}_{i} \sigma_{h}\right)=\mathcal{P}^{h}-\frac{\partial \mathcal{T}_{i}^{h}}{\partial x_{i}}+\mathcal{D}^{h}+\Theta^{h}-\epsilon^{h} .
$$


This equation contains terms for production $\mathcal{P}$, spatial transport $\mathcal{T}=\mathcal{T}^{1}+\mathcal{T}^{2}$, pressure-enthalpy correlations $\mathcal{D}$, velocity-enthalpy correlations $\Theta$, and dissipation $\epsilon$, which are given by

$$
\begin{aligned}
\mathcal{P}^{h} & \equiv-2 \widetilde{\rho} \widetilde{u_{i}^{\prime \prime} h^{\prime \prime}} \frac{\partial \widetilde{h}}{\partial x_{i}} \approx \frac{2 \mu_{\mathrm{t}}}{P r_{\mathrm{t}}}\left(\frac{\partial \widetilde{h}}{\partial x_{i}}\right)^{2}, \\
\mathcal{T}_{i}^{h 1} & \equiv \bar{\rho} \widetilde{u_{i}^{\prime \prime} h^{\prime \prime 2}} \approx-\frac{\mu_{\mathrm{t}}}{P r_{\mathrm{t}}} \frac{\partial \sigma_{h}}{\partial x_{i}}, \\
\mathcal{T}_{i}^{h 2} & \equiv \overline{2 h^{\prime \prime} q_{i}} \approx-\frac{\mu}{P r} \frac{\partial \sigma_{h}}{\partial x_{i}}, \\
\mathcal{D}^{h} & \equiv 2 \overline{h^{\prime \prime} \frac{D p}{D t}} \\
\Theta^{h} & \equiv 2 \overline{h^{\prime \prime} \tau_{i j} \frac{\partial u_{i}}{\partial x_{j}}} \\
\epsilon^{h} & \equiv-2 \frac{\partial h^{\prime \prime}}{\partial x_{i}} \approx \bar{\rho} C_{h} \frac{\sigma_{h}}{\tau_{t}} .
\end{aligned}
$$

All terms on the right-hand side of Eq. (14) require modeling. For the unclosed terms which are needed later conventional modeling approaches are given on the right sides of Eqs. (15) to (20). They are mostly based on gradient diffusion assumptions. In Eq. (20) $\tau_{t}$ is standing for a turbulence time scale and $C_{h}$ for a constant representing a turbulence to scalar time scale ratio. $\operatorname{Pr}=\mu c_{\mathrm{p}} / \lambda$ and $P r_{\mathrm{t}}=\mu_{t} c_{\mathrm{p}} / \lambda_{t}$ are molecular and turbulent Prandtl numbers, respectively.

In the case of species mass fractions the exact second moment transport equation for $\sigma_{\alpha \beta}=\widetilde{Y_{\alpha}^{\prime \prime} Y_{\beta}^{\prime \prime}}$ becomes [38]

$$
\frac{\partial}{\partial t}\left(\bar{\rho} \sigma_{\alpha \beta}\right)+\frac{\partial}{\partial x_{i}}\left(\bar{\rho} \widetilde{u}_{i} \sigma_{\alpha \beta}\right)=\mathcal{P}^{\alpha \beta}-\frac{\partial \mathcal{T}_{i}^{\alpha \beta}}{\partial x_{i}}-\epsilon^{\alpha \beta}+\mathcal{C}^{\alpha \beta}
$$

Again, all right-hand side terms have to be modeled. They are standing for production $\mathcal{P}$, spatial transport $\mathcal{T}=\mathcal{T}^{1}+\mathcal{T}^{2}$, dissipation $\epsilon$, and chemistry- 
species fluctuation interaction $\mathcal{C}$ and are given by

$$
\begin{aligned}
& \mathcal{P}^{\alpha \beta} \equiv-\bar{\rho} \widehat{u_{i}^{\prime \prime} Y_{\alpha}^{\prime \prime}} \frac{\partial \widetilde{Y}_{\beta}}{\partial x_{i}}-\bar{\rho} \widehat{u_{i}^{\prime \prime} Y_{\beta}^{\prime \prime}} \frac{\partial \widetilde{Y}_{\alpha}}{\partial x_{i}} \approx 2 \bar{\rho} D_{\mathrm{t}} \frac{\partial \widetilde{Y}_{\alpha}}{\partial x_{i}} \frac{\partial \widetilde{Y}_{\beta}}{\partial x_{i}}, \\
& \mathcal{T}_{i}^{\alpha \beta 1} \equiv \bar{\rho} \widetilde{u_{i}^{\prime \prime} Y_{\alpha}^{\prime \prime} Y_{\beta}^{\prime \prime}} \approx \frac{\mu_{\mathrm{t}}}{S c_{\mathrm{t}}} \frac{\partial \sigma_{\alpha \beta}}{\partial x_{i}} \\
& \mathcal{T}_{i}^{\alpha \beta 2} \equiv-\overline{Y_{\alpha}^{\prime \prime} \rho D_{\beta} \frac{\partial Y_{\beta}}{\partial x_{i}}}-\overline{Y_{\beta}^{\prime \prime} \rho D_{\alpha} \frac{\partial Y_{\alpha}}{\partial x_{i}}} \approx \frac{\mu}{S c} \frac{\partial \sigma_{\alpha \beta}}{\partial x_{i}}, \\
& \epsilon^{\alpha \beta} \equiv \overline{\rho D_{\beta} \frac{\partial Y_{\beta}}{\partial x_{i}} \frac{\partial Y_{\alpha}^{\prime \prime}}{\partial x_{i}}}+\overline{\rho D_{\alpha} \frac{\partial Y_{\alpha}}{\partial x_{i}} \frac{\partial Y_{\beta}^{\prime \prime}}{\partial x_{i}}} \approx-\bar{\rho} C_{\alpha \beta} \frac{\sigma_{\alpha \beta}}{\tau_{t}}, \\
& \mathcal{C}^{\alpha \beta} \equiv \overline{Y_{\alpha}^{\prime \prime} S_{\beta}}+\overline{Y_{\beta}^{\prime \prime} S_{\alpha}} \text {. }
\end{aligned}
$$

Conventional modeling approaches for some of these terms are given on the right sides of the definitions. In the modeling approaches of Eqs. (24) and (25) identical diffusion coefficients are assumed. $S c=\mu /(\rho D)$ and $S c_{\mathrm{t}}=\mu_{t} /\left(\rho D_{t}\right)$ represent molecular and turbulent Schmidt numbers, respectively. From Eq. (21) mass fraction covariance equations are obtained for $\alpha \neq \beta$ and variance equations by setting $\alpha=\beta$.

\section{Thermochemical MDF}

In the present paper the vector of thermochemical variables is given by $\boldsymbol{\Phi}=\left[\Phi_{1}, \Phi_{2}, \ldots, \Phi_{N}\right]=\left[h, Y_{1}, Y_{2}, \ldots, Y_{N-1}\right]$. The mass fraction of the last species is obtained from the normalization property. For description of the themochemical state the one-point, one-time $\operatorname{PDF} P(\boldsymbol{\Psi} ; \mathbf{x}, t)$ is used [2], where $\boldsymbol{\Psi}=\left[\Psi_{1}, \Psi_{2}, \ldots, \Psi_{N}\right]=\left[\hat{h}, \hat{Y}_{1}, \hat{Y}_{2}, \ldots, \hat{Y}_{N-1}\right]$ is the corresponding sample space vector. To solve a PDF transport equation by a stochastic particle approach, a Favre $\mathrm{PDF} \tilde{P} \equiv \rho P / \bar{\rho}$ or a $\mathrm{MDF}$

$$
F(\mathbf{\Psi} ; \mathbf{x}, t) \equiv \rho(\mathbf{\Psi} ; \mathbf{x}, t) P(\mathbf{\Psi} ; \mathbf{x}, t)
$$

is required in case of variable-density flow. A transport equation for the onepoint one-time thermochemical MDF may be derived [2] from balance Eqs. (6) 
and (7)

$$
\begin{aligned}
\frac{\partial F}{\partial t}+\frac{\partial}{\partial x_{i}}\left(\widetilde{u}_{i} F\right)+\frac{\partial}{\partial x_{i}}\left(\left\langle u_{i}^{\prime \prime} \mid \boldsymbol{\Phi}=\boldsymbol{\Psi}\right\rangle F\right) & =-\frac{\partial}{\partial \hat{Y}_{k}}\left(\frac{S_{k}}{\rho} F\right) \\
& +\mathcal{M}_{h}+\mathcal{M}_{Y}+\mathcal{S}_{h}
\end{aligned}
$$

with

$$
\begin{aligned}
\mathcal{M}_{h} & \equiv \frac{\partial}{\partial \hat{h}}\left(\left\langle\frac{\partial q_{i}}{\partial x_{i}} \mid \boldsymbol{\Phi}=\boldsymbol{\Psi}\right\rangle \frac{F}{\rho}\right), \\
\mathcal{M}_{Y} & \equiv \frac{\partial}{\partial \hat{Y}_{k}}\left(\left\langle\frac{\partial j_{k i}}{\partial x_{i}} \mid \boldsymbol{\Phi}=\boldsymbol{\Psi}\right\rangle \frac{F}{\rho}\right), \\
\mathcal{S}_{h} & \equiv-\frac{\partial}{\partial \hat{h}}\left(\left\langle\frac{\partial p}{\partial t}+u_{i} \frac{\partial p}{\partial x_{i}}+\tau_{i j} \frac{\partial u_{i}}{\partial x_{j}} \mid \boldsymbol{\Phi}=\boldsymbol{\Psi}\right\rangle \frac{F}{\rho}\right)
\end{aligned}
$$

and $k=1,2, \ldots, N-1$. All conditional expectations $(\langle\bullet \mid \mathbf{\Phi}=\mathbf{\Psi}\rangle)$ are unclosed and require modeling. The major advantage of a thermochemical MDF is the closed formulation of the strongly non-linear chemical source term (first term on the right-hand side of Eq. (28)). $\mathcal{M}_{h}$ and $\mathcal{M}_{Y}$ are standing for scalar dissipation but these terms include molecular species diffusion and heat conduction, too. $\mathcal{S}_{h}$ is caused by compressibility and viscous dissipation and is usually neglected in incompressible low Mach number flows, where temporal and spatial pressure derivatives are small, as well as the temperature increase due to viscous dissipation. However, these terms are important in high speed flows and corresponding modeling approaches are discussed later. During the MDF step the mean density and velocity (obtained from the FV solver) are frozen. Individual particle densities are calculated at the beginning of the MDF step by assumption of a constant pressure for all particles in a volume $\left(\rho=\rho\left(\boldsymbol{\Psi}, \bar{p}^{1}\right)\right)$. After the MDF simulation a new ensemble mean gas composition and temperature field is calculated using the particle-in-cell method [9] (an instantaneous averaging (IA)). In case of steady-state simulations the statistical error can be further reduced by a moving-time averaging (MTA) [39], which, in the present case, is used for mass fractions, temperature, and $\Theta_{T Y}$ (this term is needed in the FV scheme to calculate the pressure). Moreover, some practical test cases 
required an additional implicit smoothing of the MDF data $Q=\widetilde{Y}_{\alpha}, \widetilde{T}, \Theta_{T Y}$, transferred to the FV scheme, which is performed by [40, 9]

$$
\left(1-\varepsilon_{\xi} \delta_{\xi}^{2}\right)\left(1-\varepsilon_{\eta} \delta_{\eta}^{2}\right)\left(1-\varepsilon_{\zeta} \delta_{\zeta}^{2}\right) Q^{\text {new }}=Q^{\text {old }}
$$

Here $\xi, \eta, \zeta$ are the directions of the curvilinear coordinate system and $\delta^{2}$ is a discrete second-order central-difference operator. In this paper identical damping coefficients $\varepsilon_{\xi}=\varepsilon_{\eta}=\varepsilon_{\zeta}=\varepsilon$ are used. However, similar to adding artifical viscosity in case of central-difference schemes [41, 40], individual values could be advantageous in case of highly stretched grids. In simulations with multiple shock wave reflection this damping was required in order to avoid the creation of artificial shock systems due to statistical noise.

From the averaged and (in some cases) spatially damped MDF data a new pressure is calculated for the FV step using Eq. (4). The sequence of steps required for the Lagrangian particle solver as well as the coupling with the FV solver and the exchanged data are summarized on the ride side of Fig. 1. Please note, that pressure (by the equation of state) and temperature (by Newton iteration from new enthalpy and/or gas composition values) updates have to be performed several times during one iteration.

The moving-time averaging can be employed for steady-state simulations only. A local time-stepping (constant CFL number) is used in the MDF [20, 42] and the FV part of the simulation. This strongly accelerates convergence and is especially important for high speed flows. Because a compressible flow solver is employed in the FV part, the corresponding time-step size is based on $\left(u_{i}^{2}\right)^{0.5}+a$ and grid size, where $a$ is the speed of sound. Moreover, contributions from the viscous fluxes [43] are considered in a simplified way in the time-step calculation, too. This is in contrast to the Lagrangian particle solver, where the time-step size for a volume does not depend on the speed of sound. In this paper it is calculated from convective and diffusive contributions by

$$
\Delta t=\left[\frac{1}{\Delta x_{i}} \operatorname{abs}\left(\widetilde{u}_{i}+\frac{1}{\bar{\rho}} \frac{\partial}{\partial x_{i}}\left(\bar{\rho} D_{e}\right)\right)+\frac{2 D_{e}}{\left(\Delta x_{i}\right)^{2}}\right]^{-1} \cdot \mathrm{CFL},
$$


where the effective diffusivity $D_{e}=D_{t}+D$ consists of a turbulent and molecular part. Due to the different stability requirements, the FV and particle solver time-steps differ, if identical CFL numbers are used. Moreover, the implicit LUSGS solver allows much larger CFL numbers than the explicit particle solver which is limited to CFL $<1$. Thus, for steady-state simulations, the global CFL numbers of the FV and the MDF part usually differ. This means that the time-steps change from volume to volume and that additionally the FV variables and the particle properties in a cell advance differently in pseudo-time. However, in some cases it was found to be advantageous (for stability reasons) to have identical or similar time-step sizes for the FV and the MDF solver in a

volume. In the Lagrangian particle solver the local time-stepping is realized by changing the weights of particles which leave or enter a volume. This technique is described in [20] and mathematically proofed in [42].

\subsection{Partially modeled MDF transport equation}

The unclosed turbulent convection (conditional expectation on the left-hand side of Eq. (28)) is modeled by a gradient diffusion assumption as proposed by Pope [44]. This requires a turbulent diffusivity $D_{t}$ which is calculated from the eddy viscosity assuming a constant turbulent Schmidt number. As already mentioned, the unclosed term $\mathcal{S}_{h}$ is important in high speed flows and cannot be neglected. The approximation used in this paper is based on the work of Hsu et al. [45, 17]. It later has been employed in the same way by Möbus et al. $[18,20]$ for compressible RANS and similarly by Banaeizadeh et al. [46, 22] for compressible LES/FMDF simulations. By inserting these modeling approaches in Eq. (28)

$$
\begin{array}{r}
\frac{\partial F}{\partial t}+\frac{\partial}{\partial x_{i}}\left(\widetilde{u}_{i} F\right)-\frac{\partial}{\partial x_{i}}\left[\bar{\rho} D_{t} \frac{\partial}{\partial x_{i}}\left(\frac{F}{\bar{\rho}}\right)\right]=-\frac{\partial}{\partial \hat{Y}_{k}}\left(\frac{S_{k}}{\rho} F\right) \\
+\mathcal{M}_{h}+\mathcal{M}_{Y}-\frac{\partial}{\partial \hat{h}}\left(\frac{\bar{S}_{h}}{\rho} F\right)
\end{array}
$$


with

$$
\bar{S}_{h}=\frac{\partial \bar{p}}{\partial t}+\widetilde{u}_{i} \frac{\partial \bar{p}}{\partial x_{i}}+\bar{\tau}_{i j} \frac{\partial \widetilde{u}_{i}}{\partial x_{j}}+\bar{\rho} \epsilon
$$

is obtained (note that $\bar{S}_{h}$ is an approximation for the corresponding part of the exact term $S_{h}$ according to Eq. (31)). Closures for $\mathcal{M}_{h}$ and $\mathcal{M}_{Y}$ are the main subject of this paper and will be discussed later. The temporal pressure derivative in Eq. (35) is discretized by a first or second-order backward discretization, the spatial derivatives by second-order central discretizations. This is in contrast to the LES/FMDF simulations of Banaeizadeh et al. [22] who used a minmod limiter for the calculation of spatial pressure derivatives what was necessary for time-accurate simulations. The dissipation rate $\bar{\rho} \epsilon \equiv \overline{\tau_{i j} \partial u_{i}^{\prime \prime} / \partial x_{j}}$ of turbulent kinetic energy $k$ appears due to the splitting of the viscous term into a mean and a fluctuating part. $\epsilon$ is taken from the turbulence model and describes an increase in enthalpy due to dissipation of $k$. While this term is neglected in the LES/FMDF of [22] and in low speed RANS simulations, it becomes important in high speed flows.

By multiplication of Eq. (34) with $\hat{h}, \hat{Y}_{\alpha}, \hat{h}^{2}$, or $\hat{Y}_{\alpha} \hat{Y}_{\beta}$ and integration over the thermochemical space $\boldsymbol{\Psi}$ (and use of equations for $\widetilde{h}^{2}$ or $\widetilde{Y}_{\alpha} \widetilde{Y}_{\beta}$ in case of the second-order moments) first and second moment equations are derived, respectively. During this procedure $\mathcal{M}_{h}$ and $\mathcal{M}_{Y}$ are neglected because these terms will be discussed later in more detail. In this way

$$
\begin{aligned}
\frac{\partial}{\partial t}(\bar{\rho} \widetilde{h})+\frac{\partial}{\partial x_{i}}\left(\bar{\rho} \widetilde{u}_{i} \widetilde{h}-\bar{\rho} D_{t} \frac{\partial \widetilde{h}}{\partial x_{i}}\right) & =\bar{S}_{h}, \\
\frac{\partial}{\partial t}\left(\bar{\rho} \widetilde{Y}_{\alpha}\right)+\frac{\partial}{\partial x_{i}}\left(\bar{\rho} \widetilde{u}_{i} \widetilde{Y}_{\alpha}-\bar{\rho} D_{t} \frac{\partial \widetilde{Y}_{\alpha}}{\partial x_{i}}\right) & =\bar{S}_{\alpha}
\end{aligned}
$$

are obtained for the first moment equations. The gradient diffusion assumption used in the MDF equation to close turbulent convection causes correspondingly modeled terms for the Reynolds enthalpy and species fluxes. These equations may be compared with the exact transport Eqs. (10) and (11). Note that 
molecular terms $\left(\bar{q}_{i}, \bar{\tau}_{i k}, \bar{j}_{\alpha i}\right)$ do not appear in Eqs. (36) and (37) because $\mathcal{M}_{h}$ and $\mathcal{M}_{Y}$ are (for the moment) neglected. If the unclosed Reynolds fluxes are modeled with gradient type assumptions, both species equations become identical. The enthalpy equations also agree with the exception of the pressure gradient term (third term on the right-hand side of Eq. (10)). This term can be decomposed into pressure diffusion, pressure work, and pressure dilatation. The latter two appear in compressible flows only. Due to the much stronger impact of pressure on the thermochemical MDF, these terms are probably important. Modeling approaches based on averaged values could be included in Eq. (28), however, none of the modeling proposals currently available received general acceptance [47]. For this reason these terms are not considered in the MDF equation.

The agreement achieved in the first-order moments is due to correspondingly chosen modeling approaches. The question is what happens with the secondorder moments. Form the MDF transport equation (34) (without $\mathcal{M}_{h}$ and $\mathcal{M}_{Y}$ )

$$
\frac{\partial}{\partial t}\left(\bar{\rho} \sigma_{h}\right)+\frac{\partial}{\partial x_{i}}\left(\bar{\rho} \widetilde{u}_{i} \sigma_{h}-\bar{\rho} D_{t} \frac{\partial \sigma_{h}}{\partial x_{i}}\right)=-2 \bar{\rho} D_{t}\left(\frac{\partial \widetilde{h}}{\partial x_{i}}\right)^{2}+2 \bar{S}_{h} \overline{h^{\prime \prime}}
$$

follows for the enthalpy variance. A comparison with Eq. (14) shows that with the exception of the last expression in Eq. (38) all terms agree with their counterparts, if unclosed correlations are modeled by gradient like assumptions. $\mathcal{T}_{k}^{h 2}$ and $\epsilon^{h}$ are caused by molecular transport and due to negligence of $\mathcal{M}_{h}$ do not appear in Eq. (38). The last term of Eq. (38) is a very simple approximation for $\mathcal{D}^{h}$ and $\Theta_{h}$.

The transport equations for species mass fraction covariances

$$
\begin{aligned}
\frac{\partial}{\partial t}\left(\bar{\rho} \sigma_{\alpha \beta}\right)+\frac{\partial}{\partial x_{i}}\left(\bar{\rho} \widetilde{u}_{i} \sigma_{\alpha \beta}-\bar{\rho} D_{t} \frac{\partial \sigma_{\alpha \beta}}{\partial x_{i}}\right) & =-2 \bar{\rho} D_{t} \frac{\partial \widetilde{Y}_{\alpha}}{\partial x_{i}} \frac{\partial \widetilde{Y}_{\beta}}{\partial x_{i}} \\
& +\overline{Y_{\alpha}^{\prime \prime} S_{\beta}}+\overline{Y_{\beta}^{\prime \prime} S_{\alpha}}
\end{aligned}
$$


can be calculated correspondingly from the MDF equation. It agrees perfectly with the exact covariance equation (21), if gradient type approximations are used to close production $\left(\mathcal{P}^{\alpha \beta}\right)$ and turbulent transport $\left(\mathcal{T}^{\alpha \beta 1}\right)$. As the averaged chemical source term $\bar{S}_{\alpha}$ in the first moment equation, the chemistry species fluctuation correlations (last two terms or $\mathcal{C}^{\alpha \beta}$ ) appear correctly in the second moment equation and require no modeling. $\mathcal{T}^{\alpha \beta 2}$ and $\epsilon^{\alpha \beta}$ are due to molecular transport and, for the moment, are neglected in Eq. (39).

In summary, the greatest uncertainties for compressible flows are due to $\mathcal{D}^{h}$ and $\Theta^{h}$ for which better modeling approaches are needed. The modeling of $S_{h}$ (see Eq. (31)) with averaged values causes identical changes in enthalpy $\left(\bar{S}_{h}\right)$ for all particles of a volume. The model delivers correct conventionally modeled terms in the first moment equation but it is not able to reproduce the complexity of $S_{h}$ with respect to the second moments. Here, the approach of Nik et al. [23] may be more promising, because velocity and pressure correlations are treated on a particle level. However, it is also much more complex.

\section{Conditional diffusion term}

The main focus is now on different possibilities to model the conditional expectations $\mathcal{M}_{h}$ and $\mathcal{M}_{Y}$ in Eq. (34), which are standing for scalar dissipation as well as for molecular species diffusion and molecular heat conduction. Throughout this paper dissipation of fluctuations is modeled by the IEM (interaction by exchange with the mean) model [48] which is not discussed any further. In RANS approaches the molecular transport of species and enthalpy is often neglected because the turbulent transport is orders of magnitude higher. This, however, is valid for fully turbulent flows only. In general, molecular transport should be considered in RANS/URANS (unsteady RANS) simulations as well as in LES. Depending on the Reynolds number turbulent and molecular transport can be of same order of magnitude in LES [49]. In RANS simulations there may be relaminarization (e.g. in supersonic accelerating flows), laminar or weakly 
turbulent regions in jet flames with transition to a laminar surrounding [50], or, most importantly, there are viscous sublayers close to solid walls. The latter have to be resolved accurately with many two-equation turbulence models or Reynolds stress closures. In addition, differential diffusion can be important in such regions. In the following subsections two standard approaches to model molecular transport (with and without differential diffusion) are given. Next, a new technique is presented which combines the advantages of both approaches and allows a stable and accurate modeling of molecular transport even in realistic complex applications.

\subsection{Approach 1: Modeling molecular transport as drift in thermochemical space}

The following approach basically follows the technique developed by McDermott and Pope [8] for LES and later by Fiolitakis et al. [50] for RANS simulations. In this approach $\mathcal{M}_{h}$ and $\mathcal{M}_{Y}$ are modeled by

$$
\mathcal{M}_{h}+\mathcal{M}_{Y} \approx \frac{\partial}{\partial \hat{h}}\left(\frac{\partial \bar{q}_{i}}{\partial x_{i}} \frac{F}{\bar{\rho}}\right)+\frac{\partial}{\partial \hat{Y}_{k}}\left(\frac{\partial \bar{j}_{k i}}{\partial x_{i}} \frac{F}{\bar{\rho}}\right)-\frac{\partial}{\partial \Psi_{l}}\left[\frac{1}{2 \tau_{\Phi}}\left(\widetilde{\Phi}_{l}-\Psi_{l}\right) F\right]
$$

where the molecular heat transfer and molecular species diffusion are calculated from Eqs. (12) and (13), respectively. As always in this paper, the index $k$ is running over the species $k=1,2, \ldots, N-1$, while $l=1,2, \ldots, N$ is running over all thermochemical variables. The last term in Eq. (40) represents the IEM model for turbulent mixing, where $\tau_{\Phi}$ is a scalar mixing time scale calculated from $\tau_{\Phi}^{-1}=C_{\Phi} \tau_{t}^{-1}=C_{\Phi} \omega$. Here, $\omega$ is the turbulent frequency obtained from a two-equation turbulence model and $C_{\Phi}$ a turbulence to scalar time scale ratio which ist taken to be 2 in all simulations of this paper. On the particle level, McDermott and Pope [8] model molecular transport (first two terms on the right-hand side of Eq. (40)) by drifts in enthalpy and composition space (similar to the modeling of $S_{h}$ before). For any volume $\partial \bar{q}_{i} / \partial x_{i}$ and $\partial \bar{j}_{k i} / \partial x_{i}$ are constants, calculated from averaged values. In this paper the required molecular heat conductivity $\lambda=\lambda\left(\widetilde{T}, \widetilde{Y}_{1}, \cdots, \widetilde{Y}_{N}\right)$ and diffusivity 
$D_{\alpha}=D_{\alpha}\left(\widetilde{T}, \bar{p}, \widetilde{Y}_{1}, \cdots, \widetilde{Y}_{N}\right)$ are calculated from mean values, while McDermott and Pope [8] use the particle properties to calculate corresponding Favre averages $\widetilde{D}_{\alpha}$. With the present approach consistency to the FV simulation is given. The differences between both approaches are expected to be small. Thus, all particles in a volume experience identical drifts from molecular heat conduction and species diffusion but individual changes due to turbulent mixing.

Together with the remaining parts, the modeled MDF transport equation can be solved by a Lagrangian stochastic particle method [2]. The particles (indicated by the superscript $p$ ) are subject to changes in physical and thermochemical space, expressed by the following stochastic differential equations (SDEs)

$$
\begin{aligned}
x_{i}^{p}(t+d t) & =x_{i}^{p}(t)+\left[\tilde{u}_{i}+\frac{1}{\bar{\rho}} \frac{\partial}{\partial x_{i}}\left(\bar{\rho} D_{t}\right)\right] d t+\sqrt{2 D_{t}} d W_{i}, \\
h^{p}(t+d t) & =h^{p}(t)+\left[\frac{1}{\bar{\rho}} \frac{\partial \bar{q}_{i}}{\partial x_{i}}-\frac{1}{2 \tau_{\Phi}}\left(\tilde{h}-h^{p}\right)+\frac{\bar{S}_{h}}{\rho^{p}}\right] d t, \\
Y_{\alpha}^{p}(t+d t) & =Y_{\alpha}^{p}(t)+\left[\frac{1}{\bar{\rho}} \frac{\partial \bar{j}_{\alpha i}}{\partial x_{i}}-\frac{1}{2 \tau_{\Phi}}\left(\tilde{Y}_{\alpha}-Y_{\alpha}^{p}\right)+\frac{S_{\alpha}^{p}}{\rho^{p}}\right] d t,
\end{aligned}
$$

where $d W_{i}$ are the increments of an isotropic vector Wiener process. The first terms in the square brackets in Eqs. (42) and (43) describe molecular transport, the second ones scalar mixing. McDermott and Pope [8] combine molecular species transport and turbulent mixing in order to guarantee individual boundedness of the scalar field by imposing a limit on the mixing rate. If the individual boundedness of one species is violated by molecular transport, turbulent mixing of all species is increased to avoid it.

\subsubsection{First and second moments}

To investigate the impact of the modeling approach 1 on the first and second moments of $\boldsymbol{\Phi}$, Eq. (34) is extended by the modeled terms for $\mathcal{M}_{h}$ and $\mathcal{M}_{Y}$ according to Eq. (40). Only results stemming from these terms are discussed in 
the following. The additional right-hand side contributions from $\mathcal{M}_{h}$ and $\mathcal{M}_{Y}$ become

$$
\begin{aligned}
\frac{\partial}{\partial t}(\bar{\rho} \widetilde{h}) & =\ldots-\frac{\partial \bar{q}_{i}}{\partial x_{i}} \\
\frac{\partial}{\partial t}\left(\bar{\rho} \widetilde{Y}_{\alpha}\right) & =\ldots-\frac{\partial \bar{j}_{\alpha i}}{\partial x_{i}}
\end{aligned}
$$

Note, that these contributions to the first moments are exclusively from molecular transport and not from turbulent mixing (IEM model). A comparison with Eqs. (10) and (11) shows, that the modeling approach 1 achieves identical terms as conventional closures for the first order moment equations. In a similar way the right-hand side contributions of $\mathcal{M}_{h}$ and $\mathcal{M}_{Y}$ to the second-order moments are calculated

$$
\begin{aligned}
\frac{\partial}{\partial t}\left(\bar{\rho} \sigma_{h}\right) & =\ldots-\bar{\rho} \frac{\sigma_{h}}{\tau_{\Phi}}, \\
\frac{\partial}{\partial t}\left(\bar{\rho} \sigma_{\alpha \beta}\right) & =\ldots-\bar{\rho} \frac{\sigma_{\alpha \beta}}{\tau_{\Phi}} .
\end{aligned}
$$

These terms now exclusively result from turbulent mixing. The molecular transport approach of Eq. (40) has no impact on the second-order moments. However, a comparison with Eqs. (14) and (21) shows, that molecular transport also causes the terms $\mathcal{T}^{h 2}$ and $\mathcal{T}^{\alpha \beta 2}$, which describe a spatial transport of variance. These terms are not reproduced by the present model [8].

\subsubsection{Advantages and disadvantages of approach 1}

The great advantage of Eq. (40) to model molecular transport is the possibility to account for differential diffusion and thus non-equal Prandtl and Schmidt numbers. On the other hand there are the described problems with individual boundedness which can be solved by accelerating turbulent mixing [8] or by a simple clipping [50]. However, there may be numerical problems, too, if molecular heat conduction and species diffusion are treated by constant drifts in thermochemical space. In case of steady-state simulations and local timestepping (constant CFL number) [20,42] volumes may run out of particles, 

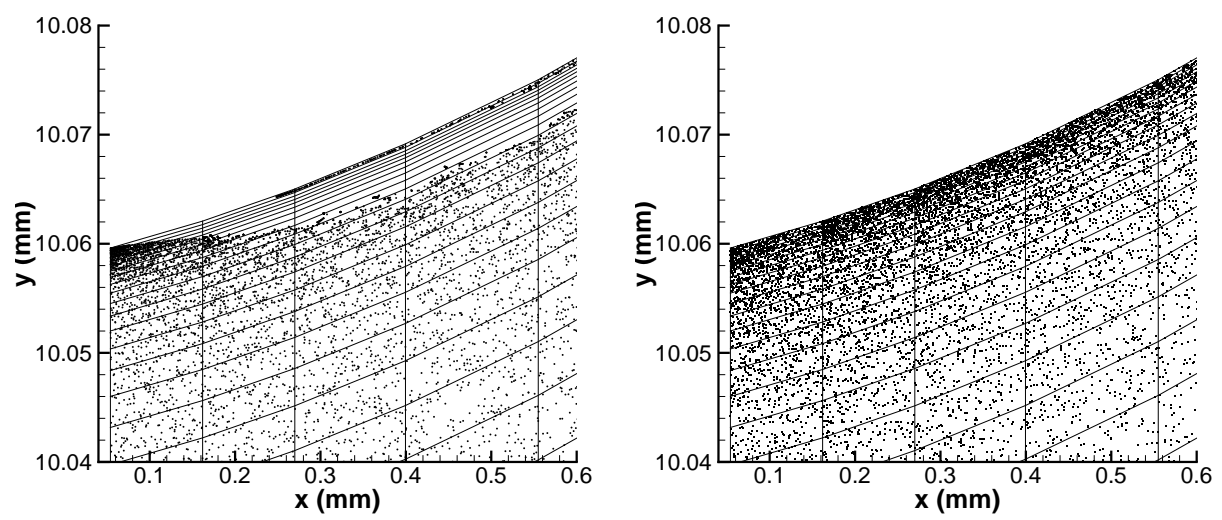

Figure 2: Particle positions (dots) and computational grid (lines) for a nozzle flow simulation in an extension close to the upper wall. Left side Wiener process with $D_{t}$ only (approach 1 ), right side with $D_{e}=D_{t}+D$ (approaches 2 and 3 ).

e.g. in the viscous sublayer. Please note, that this problem arises due to nonphysical intermediate states caused by the local time-stepping before reaching a statistically steady-state solution. If $D_{t}$ becomes zero in laminar parts of the flow due to $\mu_{t} \rightarrow 0$, the stochastic contribution from the Wiener process to the particle movement (last term in Eq. (41)) vanishes. This has the effect that all particles in volumes with $\mu_{t} \approx 0$ (e.g. in the viscous sublayer close to a solid wall) move with identical velocity, if the local constant mean estimate (LCME) [51] is used to initialize the particles with FV velocity data. As a consequence volumes may run empty if the common flow direction is out of a volume and no particles enter on the opposite side, for example because there is a solid wall. This problem for $\mu_{t} \approx 0$ regions (laminar zones) often occurs in volumes with high cell aspect ratios or if the instantaneous flow direction does not agree with the curvilinear grid. Examples for both cases are given on the left sides of Figs. 2 and 3 . According to approach 1 , only $D_{t}$ is used in the stochastic part of the Wiener process. Figure 2 is from a nozzle flow simulation with sonic inlet at the nozzle throat on the left side. The picture shows an extension of the first part of the grid close to the upper wall. The lines indicate computational volumes, 

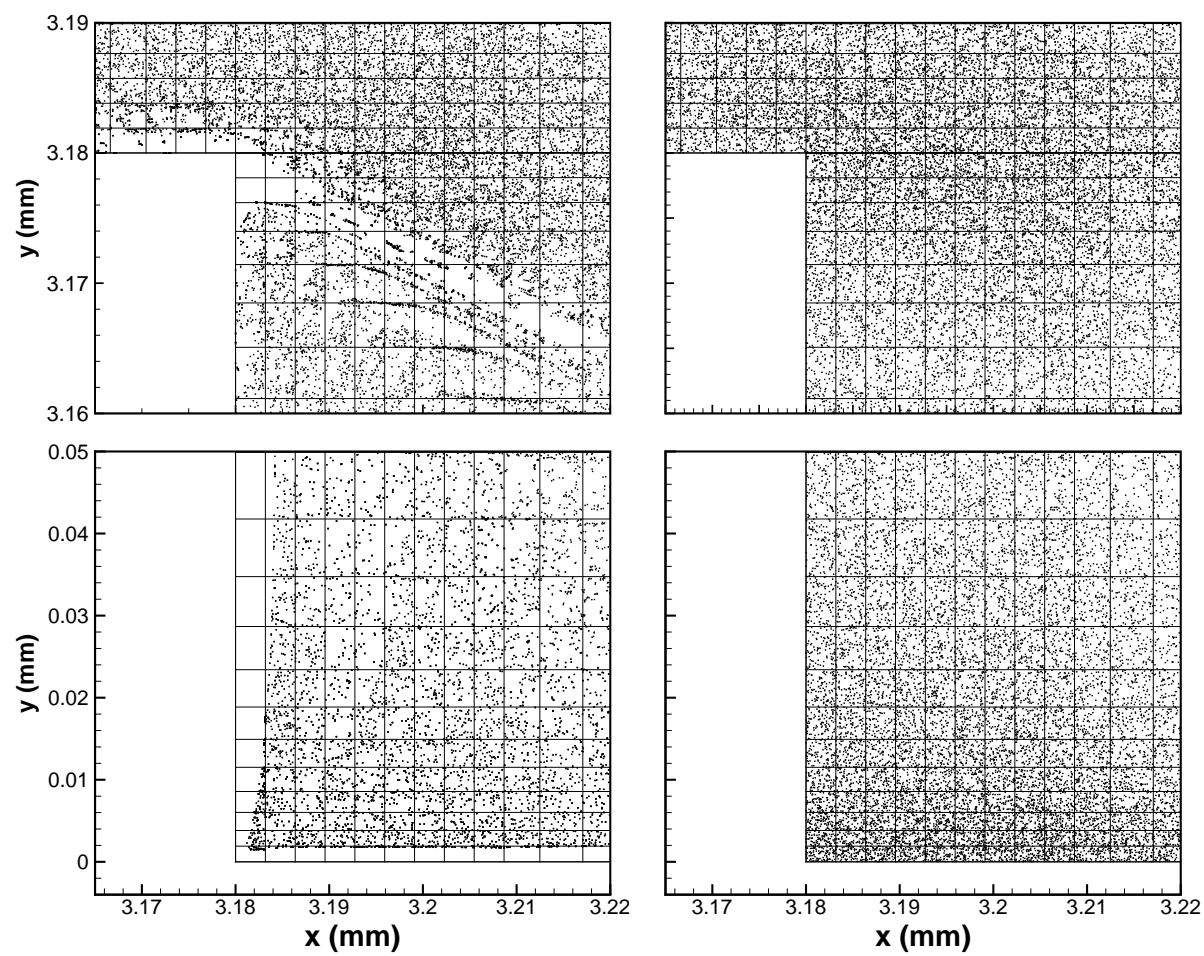

Figure 3: Particle positions (dots) and computational grid (lines) for a backward facing step simulation in an extension close to the upper corner of the step (upper figures) and at the lower corner (lower figures) after the step. Left side Wiener process with $D_{t}$ only (approach 1), right side with $D_{e}=D+D_{t}$ (approaches 2 and 3).

the dots particle positions at a given time-step. Note that this figure is not to scale but highly stretched in $y$-direction. The cell aspect ratio of the first volumes adjacent to the wall is approximately 2800. Due to the axial inflow at the nozzle throat and the slowly developing flow field, a large number of volumes runs out of particles. This happens in many practical applications with high cell aspect ratios and turbulence models which resolve the viscous sublayer. A second example is the supersonic backward facing step flow from Sect. 6.3 which demonstrates, that the described problem is not limited to highly stretched boundary layer grids. Figure 3 shows extensions of the region directly at the upper corner of the backward facing step (upper figures) and at the lower 
corner downstream of the step (lower figures). The complete configuration is plotted in Fig. 7. The cell aspect ratios in this case are very low. The incoming viscous sublayer with $\mu_{t} \approx 0$ experiences a sudden change in flow direction at the step. The particles follow the main flow direction and a large number of volumes becomes empty. No thermochemical data is available in these volumes and without special fixes, the simulation is stalled. In case of time-accurate simulations this problem should not occur because at any time there always has to be mass in every volume. The situation may be slightly better, if the velocities from the $\mathrm{FV}$ simulation are interpolated to the particle positions. In this case, however, the problem arises, that particles accumulate close to solid walls and are not able to leave due to extremely low flow velocities. For these reasons a different approach is required.

5.2. Approach 2: Modeling molecular transport as part of the stochastic Wiener process

If identical diffusion coefficients are assumed for all species and Prandtl and Schmidt numbers are identical (Lewis number of one) the averaged molecular heat and species fluxes take similar forms and instead of Eqs. (12) and (13)

$$
\begin{aligned}
\breve{q}_{i} & =-\frac{\mu}{\operatorname{Pr}} \frac{\partial \tilde{h}}{\partial x_{i}}=-\frac{\mu}{S c} \frac{\partial \tilde{h}}{\partial x_{i}}=-\bar{\rho} D \frac{\partial \tilde{h}}{\partial x_{i}}, \\
\breve{j}_{\alpha i} & =-\bar{\rho} D \frac{\partial \tilde{Y}_{\alpha}}{\partial x_{i}}
\end{aligned}
$$

is obtained. Note that the correction term required in Eq. (13) disappears in case of equal diffusion coefficients. There are different possibilities to calculate the average molecular diffusion coefficient $D$. In this paper a mass fraction weighted average of the individual molecular diffusion coefficients is chosen $D=$ $\sum_{\alpha} \widetilde{Y}_{\alpha} D_{\alpha}$. Alternatively, $D=\lambda /\left(\bar{\rho} c_{\mathrm{p}}\right)$ may be used. Another possibility is the calculation from $\mu$ using a chosen Schmidt number as given in Eq. (48). Minor differences are expected in $D$ stemming from the different approaches.

Under the simplifications given above $\mathcal{M}_{h}$ and $\mathcal{M}_{Y}$ from Eqs. (29) and (30) 
may by summarized to

$$
\mathcal{M}_{h}+\mathcal{M}_{Y}=\frac{\partial}{\partial \Psi_{l}}\left[\left\langle\frac{\partial}{\partial x_{i}}\left(\rho D \frac{\partial \Phi_{l}}{\partial x_{i}}\right) \mid \boldsymbol{\Phi}=\boldsymbol{\Psi}\right\rangle \frac{F}{\rho}\right]
$$

In the modeling approach 2 this conditional expectation is modeled as [20]

$$
\mathcal{M}_{h}+\mathcal{M}_{Y} \approx \frac{\partial}{\partial x_{i}}\left[\bar{\rho} D \frac{\partial}{\partial x_{i}}\left(\frac{F}{\bar{\rho}}\right)\right]-\frac{\partial}{\partial \Psi_{l}}\left[\frac{1}{2 \tau_{g}}\left(\widetilde{\Phi}_{l}-\Psi_{l}\right) F\right]
$$

where, again, the IEM model is used for turbulent mixing. In this equation $\tau_{\Phi}$ is replaced by a time scale $\tau_{g}$. The reason for it is given later. In the modeling approach 2, molecular transport (first term on the right-hand side of Eq. (51)) has the same form (with $D$ instead of $D_{t}$ ) as turbulent transport in the MDF transport equation. Thus, on a particle level, both terms may be modeled together. In this way the effective diffusivity $D_{e}=D_{t}+D$ appears in the stochastic Wiener process as well as in the drift term. The latter one is identical for all particles in a volume. Combined with the remaining parts of the modeled MDF equation the particle properties now change according to the SDEs

$$
\begin{aligned}
x_{i}^{p}(t+d t) & =x_{i}^{p}(t)+\left[\tilde{u}_{i}+\frac{1}{\bar{\rho}} \frac{\partial}{\partial x_{i}}\left(\bar{\rho} D_{e}\right)\right] d t+\sqrt{2 D_{e}} d W_{i} \\
h^{p}(t+d t) & =h^{p}(t)-\left[\frac{1}{2 \tau_{g}}\left(\tilde{h}-h^{p}\right)-\frac{\bar{S}_{h}}{\rho^{p}}\right] d t \\
Y_{\alpha}^{p}(t+d t) & =Y_{\alpha}^{p}(t)-\left[\frac{1}{2 \tau_{g}}\left(\tilde{Y}_{\alpha}-Y_{\alpha}^{p}\right)-\frac{S_{\alpha}^{p}}{\rho^{p}}\right] d t .
\end{aligned}
$$

Due to the modeling of molecular diffusion by stochastic particle movement there is one single free parameter $(D)$ for enthalpy and species only. Thus differential diffusion and non-equal Prandtl and Schmidt numbers cannot be realized with this technique. 


\subsubsection{First and second moments}

Using the modeling approach (51) in the modeled MDF Eq. (34) the additional contributions from $\mathcal{M}_{h}$ and $\mathcal{M}_{Y}$ to the right-hand sides of the first-order moment equations

$$
\begin{aligned}
\frac{\partial}{\partial t}(\bar{\rho} \widetilde{h}) & =\ldots-\frac{\partial \breve{q}_{i}}{\partial x_{i}}, \\
\frac{\partial}{\partial t}\left(\bar{\rho} \widetilde{Y}_{\alpha}\right) & =\ldots-\frac{\partial \breve{j}_{\alpha i}}{\partial x_{i}}
\end{aligned}
$$

are constant Prandtl and Schmidt number approximations of approach 1 (see Eqs. (44) and (45)). Moreover, the first moments agree with the corresponding terms of the standard first-order moment equations (10) and (11), if identical assumptions and modeling approaches are taken $\left(D_{\alpha}=D, \operatorname{Pr}=S c\right)$. Differences to approach 1 occur, if the contributions to the right-hand side of the second-order moments are calculated which become

$$
\begin{aligned}
\frac{\partial}{\partial t}\left(\bar{\rho} \sigma_{h}\right) & =\ldots+\frac{\partial}{\partial x_{i}}\left(\bar{\rho} D \frac{\partial \sigma_{h}}{\partial x_{i}}\right)+2 \bar{\rho} D\left(\frac{\partial \tilde{h}}{\partial x_{i}}\right)^{2}-\bar{\rho} \frac{\sigma_{h}}{\tau_{\Phi}}, \\
\frac{\partial}{\partial t}\left(\bar{\rho} \sigma_{\alpha \beta}\right) & =\ldots+\frac{\partial}{\partial x_{i}}\left(\bar{\rho} D \frac{\partial \sigma_{\alpha \beta}}{\partial x_{i}}\right)+2 \bar{\rho} D \frac{\partial \tilde{Y}_{\alpha}}{\partial x_{i}} \frac{\partial \tilde{Y}_{\beta}}{\partial x_{i}}-\bar{\rho} \frac{\sigma_{\alpha \beta}}{\tau_{\Phi}},
\end{aligned}
$$

if $\tau_{g}=\tau_{\Phi}$ is used. The last term of each equation results from the turbulent mixing model while the first two terms are caused by molecular transport. The latter ones do not appear in approach 1. A comparison with the exact Eqs. (14) and (21) shows, that each first term is correct on a modeled level and describes transport of variance in physical space. The second terms however, are unwanted non-physical production terms, which are termed spurious production in Ref. [52]. In fully turbulent regions $\left(\mu_{t} \gg \mu\right)$ this production of variance is small compared to the turbulent production terms $\mathcal{P}^{h}$ and $\mathcal{P}^{\alpha \beta}$ (see Eqs. (15) and (22), respectively). Therefore, as long as the flow is fully turbulent, approach 2 may be used. Problems with non-physical variance production are limited to laminar regions, regions with low turbulence levels, and the viscous sublayer. Approaching the wall, $\omega \rightarrow \infty$ and $\tau_{\Phi} \rightarrow 0$ cause a strong reduction 
of variance due to turbulent mixing. On the other hand, there may be large enthalpy gradients in case of cooled walls.

\subsubsection{Modified mixing time scale}

A simple possibility to correct the non-physical variance production terms and to achieve more accurate second-order moments is to modify the time scale of the IEM model. A similar approach has been used by Pozorski and Minier [53] for a single variable in a temperature PDF equation. As shown before, the IEM model keeps the first moment constant and causes a reduction of variance. In order to correct the non-physical molecular production terms, a new time scale $\tau_{g}$ is introduced by

$$
\frac{1}{\tau_{g}}=\frac{1}{\tau_{m}}+\frac{1}{\tau_{\Phi}}
$$

with

$$
\frac{1}{\tau_{m}}=2 \frac{D}{\sigma_{\Phi}}\left(\frac{\partial \tilde{\Phi}}{\partial x_{i}}\right)^{2}
$$

in case of a single scalar $\Phi\left(\sigma_{\Phi}\right.$ is the variance of $\left.\Phi\right)$. Using the new time scale $\tau_{g}$ instead of $\tau_{\Phi}$ in the IEM model (as done in Eqs. (53) and (54)) causes an additional destruction term (antidiffusion in scalar phase space), which, in the statistical limit, is identical to the unwanted non-physical production but with opposite sign. In contrast to [53] multiple scalars have to be treated here. While individual second-order momen production terms are caused by approach 2 for any variable combination $\alpha, \beta$, a single common destruction time scale $\tau_{m}$ can be introduced in the IEM model for all variances and covariances (enthalpy and species) only. Thus, for multi-scalar problems, the following approach is only approximately able to cancel out the non-physical second-order production terms. While for the enthalpy equation alone an exact destruction time scale

$$
\frac{1}{\tau_{h}}=\frac{2 D}{\sigma_{h}}\left(\frac{\partial \tilde{h}}{\partial x_{i}}\right)^{2},
$$


can be defined, an averaged value

$$
\frac{1}{\tau_{Y}}=\frac{2 D}{\sum_{\alpha=1}^{N} \sigma_{\alpha \alpha}} \sum_{\alpha=1}^{N}\left(\frac{\partial \tilde{Y}_{\alpha}}{\partial x_{i}}\right)^{2}
$$

is proposed for the species. For simplicity this approach is based on variances only. The required values $\sigma_{h}$ and $\sigma_{\alpha \alpha}$ are calculated for any volume from the particle properties. In this paper instantaneous averages are used but if a further reduction of their statistical error is required, a moving-time averaging [39] can be employed for $\sigma_{h}$ and $\sigma_{\alpha \alpha}$ in case of steady-state simulations. To avoid division by zero a lower limit for the variances must be chosen. Next, the maximum

$$
\frac{1}{\tau_{m}}=\max \left(\frac{1}{\tau_{h}}, \frac{1}{\tau_{Y}}\right)
$$

is taken to account for cases, where either species or enthalpy variances are dominating.

The described technique works very well in most cases. However, problems arise when strong gradients occur, e.g. in the first volumes downstream of a splitter plate which separates streams of different gas composition (see test cases of Sects. 6.4, 6.5, and 6.6). The large species gradients which exist locally in such regions cause large values of $1 / \tau_{m}$ and thus a rapid mixing. The problem is, that in the IEM model $\tau_{m}$ causes a numerical diffusion which scales with $\Delta x^{2} / \tau_{m}$, where $\Delta x$ is the grid spacing. Hence, a fast mixing in thermochemical space due to large values of $1 / \tau_{m}$ may induce numerical errors in physical space, where the scalar transport can become too diffusive (of course $\tau_{\Phi}$ causes corresponding errors in the IEM model but the focus is here on $\tau_{m}$ ). Therefore, a limitation is introduced

$$
\frac{1}{\tau_{m}}=\min \left(\frac{1}{\tau_{m}}, C_{m} \frac{1}{\tau_{c}}\right)
$$

which limits the degree of possible mixing due to $\tau_{m}$ in a volume. In this equation $\tau_{c}=l_{c} /\left(\widetilde{u}_{i}^{2}\right)^{0.5}$ is a mean flow through time through the volume with $l_{c}$ being a characteristic length of the cell. $C_{m}$ is a constant of the order of one. 
As will be shown later in practical investigations, $C_{m}=1$ is a good compromise. With this value the non-physically produced variance of a single scalar is reduced by $66 \%$ when the particles cross the volume. A value of $C_{m}=4$ already achieves $98 \%$ reduction but causes a more diffusive behavior, too. The impact of $C_{m}$ on the numerical results is discussed in detail later.

\subsubsection{Advantages and disadvantages of approach 2}

By modeling molecular transport in the Wiener process, no problems with boundedness of species mass fractions occur. Moreover, a stochastic movement of particles takes place everywhere, even in laminar regions such as the viscous sublayer. As a result, volumes do not run out of particles in constant CFL number simulations. Figures 2 and 3 show this on their right sides. They are simulated with approach 3 but approach 2 achieves identical results, because in both cases differential diffusion is not important. These examples clearly demonstrate the numerical advantages of approach 2 for practical applications and especially high speed flows. Note however, that molecular diffusion in the Wiener process is not inherently essential in MDF methods. Further, compared to approach 1, the molecular transport of variance is included correctly. Moreover, the presented modification of the mixing time scale of the IEM model reduces the unwanted non-physical variance production. A more detailed discussion concerning this point is given in the next section. Thus, the main disadvantage of approach 2 is the missing possibility to account for differential diffusion.

\subsection{Approach 3: Combination of approaches 1 and 2}

To overcome the disadvantage of approach 2 but retain its good numerical stability, a combination with approach 1 is introduced. First the molecular heat 
and species fluxes are divided into two parts

$$
\begin{aligned}
\bar{q}_{i} & =\breve{q}_{i}+\Delta \bar{q}_{i}, \\
\bar{j}_{\alpha i} & =\breve{j}_{\alpha i}+\Delta \bar{j}_{\alpha i} .
\end{aligned}
$$

Using Eqs. (12), (13), (48), and (49)

$$
\begin{aligned}
\Delta \bar{q}_{i} & =-\left(\lambda-\bar{\rho} c_{p} D\right) \frac{\partial \tilde{T}}{\partial x_{i}}+\sum_{\alpha=1}^{N} \tilde{h}_{\alpha} \Delta \bar{j}_{\alpha i}, \\
\Delta \bar{j}_{\alpha i} & =-\bar{\rho}\left(D_{\alpha}-D\right) \frac{\partial \tilde{Y}_{\alpha}}{\partial x_{i}}+\bar{\rho} \tilde{Y}_{\alpha} \sum_{\beta=1}^{N} D_{\beta} \frac{\partial \tilde{Y}_{\beta}}{\partial x_{i}}
\end{aligned}
$$

can be calculated for any volume based on averaged data. In this way the molecular heat and species transport with individual conductivity and diffusion coefficients $\left(\bar{q}_{i}\right.$ and $\left.\bar{j}_{\alpha i}\right)$ is divided into a part with equal diffusivity and Lewis number one $\left(\breve{q}_{i}\right.$ and $\left.\breve{j}_{\alpha i}\right)$ and individual correction terms $\left(\Delta \bar{q}_{i}\right.$ and $\left.\Delta \bar{j}_{\alpha i}\right)$. Based on this division, the following modeling approach is proposed to model molecular heat and species transport and scalar mixing

$$
\begin{aligned}
\mathcal{M}_{h}+\mathcal{M}_{Y} \approx & \frac{\partial}{\partial x_{i}}\left[\bar{\rho} D \frac{\partial}{\partial x_{i}}\left(\frac{F}{\bar{\rho}}\right)\right]+\frac{\partial}{\partial \hat{h}}\left(\frac{\partial \Delta \bar{q}_{i}}{\partial x_{i}} \frac{F}{\bar{\rho}}\right)+\frac{\partial}{\partial \hat{Y}_{k}}\left(\frac{\partial \Delta \bar{j}_{k i}}{\partial x_{i}} \frac{F}{\bar{\rho}}\right) \\
& -\frac{\partial}{\partial \Psi_{l}}\left[\frac{1}{2 \tau_{\Phi}}\left(\widetilde{\Phi}_{l}-\Psi_{l}\right) F\right] .
\end{aligned}
$$

From the three terms describing molecular transport (first three terms on the right-hand side) the first one contributes to the stochastic Wiener process. The next two terms represent identical shifts in thermochemical space for all particles in a volume due to differential diffusion. If approach 3 is used in the modeled MDF Eq. (34), it can be solved by the following SDEs

$$
\begin{aligned}
x_{i}^{p}(t+d t) & =x_{i}^{p}(t)+\left[\tilde{u}_{i}+\frac{1}{\bar{\rho}} \frac{\partial}{\partial x_{i}}\left(\bar{\rho} D_{e}\right)\right] d t+\sqrt{2 D_{e}} d W_{i} \\
h^{p}(t+d t) & =h^{p}(t)+\left[\frac{1}{\bar{\rho}} \frac{\partial \Delta \bar{q}_{i}}{\partial x_{i}}-\frac{1}{2 \tau_{g}}\left(\tilde{h}-h^{p}\right)+\frac{\bar{S}_{h}}{\rho^{p}}\right] d t \\
Y_{\alpha}^{p}(t+d t) & =h^{p}(t)+\left[\frac{1}{\bar{\rho}} \frac{\partial \Delta \bar{j}_{\alpha i}}{\partial x_{i}}-\frac{1}{2 \tau_{g}}\left(\tilde{Y}_{\alpha}-Y_{\alpha}^{p}\right)+\frac{S_{\alpha}^{p}}{\rho^{p}}\right] d t .
\end{aligned}
$$


As before in approach $2, \tau_{g}$ is used in the IEM model instead of $\tau_{\Phi}$ to reduce the non-physical variance production. The shifts in composition space $\Delta \bar{j}_{\alpha i}$ may induce problems with species boundedness. However, these problems are expected to be smaller than in case of approach 1, because significant parts of molecular diffusion are covered by the Wiener process. Thus a simple clipping and a normalization of species mass fractions is performed after the fractional step diffusion.

\subsubsection{First and second moments}

Inserting approach (69) to model $\mathcal{M}_{h}+\mathcal{M}_{Y}$ in the MDF Eq. (34), the following contributions to the right-hand sides of the first and second moment equations are obtained

$$
\begin{aligned}
\frac{\partial}{\partial t}(\bar{\rho} \widetilde{h}) & =\ldots-\frac{\partial \bar{q}_{i}}{\partial x_{i}} \\
\frac{\partial}{\partial t}\left(\bar{\rho} \widetilde{Y}_{\alpha}\right) & =\ldots-\frac{\partial \bar{j}_{\alpha i}}{\partial x_{i}} \\
\frac{\partial}{\partial t}\left(\bar{\rho} \sigma_{h}\right) & =\ldots+\frac{\partial}{\partial x_{i}}\left(\bar{\rho} D \frac{\partial \sigma_{h}}{\partial x_{i}}\right)-\bar{\rho} \frac{\sigma_{h}}{\tau_{\Phi}}+\bar{\rho} \sigma_{h}\left(\frac{1}{\tau_{h}}-\frac{1}{\tau_{m}}\right) \\
\frac{\partial}{\partial t}\left(\bar{\rho} \sigma_{\alpha \beta}\right) & =\ldots+\frac{\partial}{\partial x_{i}}\left(\bar{\rho} D \frac{\partial \sigma_{\alpha \beta}}{\partial x_{i}}\right)-\bar{\rho} \frac{\sigma_{\alpha \beta}}{\tau_{\Phi}}+\bar{\rho} \sigma_{\alpha \beta}\left(\frac{1}{\tau_{\alpha \beta}}-\frac{1}{\tau_{m}}\right)
\end{aligned}
$$

with $\tau_{h}$ according to Eq. (61) and

$$
\frac{1}{\tau_{\alpha \beta}}=\frac{2 D}{\sigma_{\alpha \beta}} \frac{\partial \tilde{Y}_{\alpha}}{\partial x_{i}} \frac{\partial \tilde{Y}_{\beta}}{\partial x_{i}}
$$

The last terms in Eqs. (75) and (76) are due to the spurious variance production $\left(1 / \tau_{h}\right.$ and $\left.1 / \tau_{\alpha \beta}\right)$ and the newly introduced destruction term $\left(1 / \tau_{m}\right)$. In case of a single scalar, the non-physical variance production can be completely removed (e.g. with $\tau_{m}=\tau_{h}$ in case of the enthalpy equation). However, for multiscalar problems, the IEM model provides one single free parameter $\left(\tau_{m}\right)$ for all variances and covariances only. Hence, the chosen averaged time scale $\tau_{m}$ (see 
Eq. (64)) is only approximately able to cancel out the non-physical variance and covariance production. Nevertheless the described method works well as will be shown later in practical applications. Moreover, its use is supported by the following analysis for idealized conditions. In the limit $\mu_{t} \rightarrow 0$ (only then the spurious production terms become important), for non-reactive flows, and assuming local equilibrium (spatial transport is neglected) the enthalpy and species variance equations reduce to

$$
\begin{aligned}
2 \bar{\rho} D\left(\frac{\partial \tilde{h}}{\partial x_{i}}\right)^{2} & =\bar{\rho} C_{h} \frac{\sigma_{h}}{\tau_{t}}, \\
2 \bar{\rho} D \frac{\partial \tilde{Y}_{\alpha}}{\partial x_{i}} \frac{\partial \tilde{Y}_{\beta}}{\partial x_{i}} & =\bar{\rho} C_{\alpha \beta} \frac{\sigma_{\alpha \beta}}{\tau_{t}} .
\end{aligned}
$$

For these idealized conditions and assuming identical turbulence to scalar time scale ratios $C_{\alpha \beta}=C_{h}=C_{\Phi}$, the following algebraic conditions

$$
\begin{aligned}
\sigma_{h} & =2 D \frac{\tau_{t}}{C_{\Phi}}\left(\frac{\partial \tilde{h}}{\partial x_{i}}\right)^{2}, \\
\sigma_{\alpha \beta} & =2 D \frac{\tau_{t}}{C_{\Phi}} \frac{\partial \tilde{Y}_{\alpha}}{\partial x_{i}} \frac{\partial \tilde{Y}_{\beta}}{\partial x_{i}}
\end{aligned}
$$

are obtained for the variances and covariances. Inserting these values into Eq. (61) and (77) the spurious variance production time scales

$$
\frac{1}{\tau_{h}}=\frac{1}{\tau_{\alpha \beta}}=\frac{C_{\Phi}}{\tau_{t}}
$$

become identical for all variables. Using the same algebraic relations (80) and (81) for the calculation of the corresponding destruction term time scale $\tau_{m}$ by Eq. (63) (without the limitation from Eq. (64)), an identical time scale

$$
\frac{1}{\tau_{m}}=\frac{C_{\Phi}}{\tau_{t}}
$$

is obtained. Thus, under the above given idealized local equilibrium conditions, the spurious variance and covariance production terms could be completely removed and the last terms in Eqs. (75) and (76) vanish. This, however, is not 
valid in general, especially not for cases with chemistry and strong spatial transport.

\subsubsection{Advantages and disadvantages of approach 3}

Approach 3 combines the advantages from approach 1 and 2. The stochastic movement of the particles in laminar regions allows a numerically stable simulation without volumes running out of particles in constant CFL number simulations. Differential diffusion is considered and the first-order moments are predicted correctly. Moreover, compared to approach 1, the molecular transport of variance is correctly included. Nevertheless, there still is the problem of spurious variance production in case of multi-scalar problems. Another question is the choice of the limitation constant $C_{m}$ in Eq. (64) and the occurrence of numerical diffusion in case of short time scales $\tau_{m}$ in the IEM model. These topics are discussed further on the basis of practical investigations in the next section.

\section{Results and Discussion}

In order to demonstrate the correctness and accuracy of the described techniques if applied to compressible flows, a number of simulations are performed with different levels of complexity. To enable a step by step investigation of isolated effects some simple (partially purely academic) test cases are chosen. Effects like viscous work, the modeling of pressure gradients, differential diffusion, or the heating of the boundary layer in high speed flows are independent from combustion. Thus, many test cases are non-reactive. In these cases a comparison with standard FV simulations allows to check the consistency of the implementation and the correctness of the hybrid MDF/FV simulation. At the end of this section a supersonic mixing channel and model rocket combustor

will be presented. If not stated otherwise, all simulations use approach 3 (see Sect. 5.3) and approximately 100 particles per volume. The particle number is 
controlled by particle splitting and fusion using individual particle masses [12]. For this purpose a preferred weight $\bar{m}$ is calculated for any volume by dividing the total particle mass in the volume through the desired number of particles. If the ratio between the actual particle weight and the preferred weight is higher or smaller than a certain factor $c$ (in all simulations of this paper this factor is $c=0.5$ ), than particle splitting or fusion is performed. Thus all particles with $m^{p} \geq \bar{m} / c$ are replaced by $\operatorname{int}\left(m^{p} / \bar{m}\right)$ particles with identical properties and new calculated weights. On the other hand, the weight of all particles with $m^{p} \leq \bar{m} c$ is increased to $\bar{m}$ with probability $m^{p} / \bar{m}$ or the particle is extinguished with probability $1-m^{p} / \bar{m}$.

\subsection{Mach 4.5 flow over an adiabatic flat plate}

In order to assess the effect of heating of the fluid in a high speed boundary layer with adiabatic wall, the experiment of Mabey et al. $[54,55]$ is chosen. Due to the high flow velocity (Mach 4.5) viscous dissipation becomes important close to the solid wall. Thus, the modeling approach from Eq. (35) (without the temporal pressure derivative term) can be validated. For this the viscous sublayer must be resolved by the computational grid $\left(y^{+}<1\right)$ due to the requirements of the low Reynolds number $q-\omega$ turbulence closure [30]. The turbulence model has to predict the transition from a fully turbulent exterior flow into the laminar sublayer close to the wall. The corresponding processes on the energy side (temperature boundary layer) have to be captured by the Lagrangian particle solver. If a particle hits the wall it is reflected and maintains its temperature. Freestream temperature and pressure are $62 \mathrm{~K}$ and $3.2 \mathrm{kPa}$, respectively. In the experiment, a roughness band was used to move transition towards the leading edge of the plate and thus to increase the length of turbulent flow. All calculations are performed through transition and boundary layer profiles are compared with experimental ones at identical displacement thicknesses. The calculations use constant inflow conditions and a turbulence intensity of $1 \%$. The computational grid consist of $192 \cdot 80$ volumes for a length of $1.62 \mathrm{~m}$ in $x$ - 

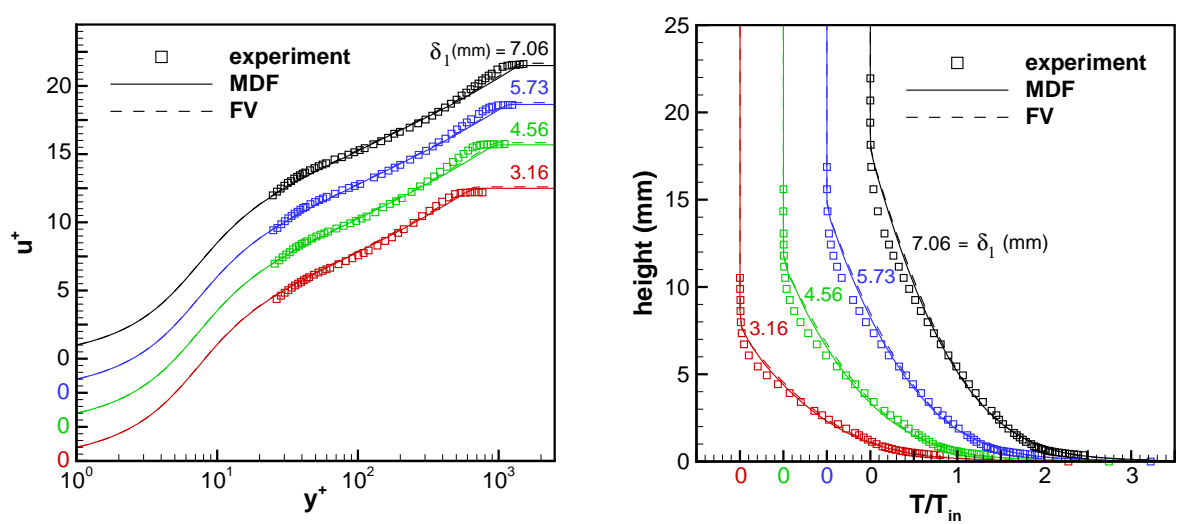

Figure 4: Normalized measured [54] and calculated (FV solver and hybrid MDF/FV scheme) velocity $\left(u^{+}=u / u_{\tau}\right.$, left side) and temperature (right side) profiles at different displacement thicknesses of a turbulent Mach 4.5 boundary layer.

and $0.86 \mathrm{~m}$ in $y$-direction. The grid is highly refined close to the wall and the distance of the first cell centers to the wall is $0.5 \cdot 10^{-6} \mathrm{~m}$. Thus, all $y^{+}$-values of near wall cell centers are below 0.32 . The resulting cell aspect ratio is up to 2400, which is quite extreme for a Lagrangian particle solver. Figure 4 shows normalized calculated velocity (left side) and temperature (right side) profiles at four different displacement thicknesses $\delta_{1}$ of the boundary layer (different $x$-positions) in comparison with the experiment. A blow up of the temperature profile close to the wall is given in Fig. 5. For a better representation of the results, the origin of the $x$ - or $y$-axis is shifted for every profile. The same technique is used in some of the following figures, too. Both velocity and temperature profiles agree very well with the experimental data. The hybrid MDF/FV results (solid lines) in Fig. 4 are nearly identical to the pure FV profiles (dashed lines) and hard to distinguish. Please note that MDF solution in the figures always means a hybrid scheme with MDF for the thermochemical variables and a standard FV scheme for the remaining ones. The results show, that the viscous dissipation term in the energy equation is well reproduced on the particle level. Moreover, laminarization in the viscous sublayer is modeled 


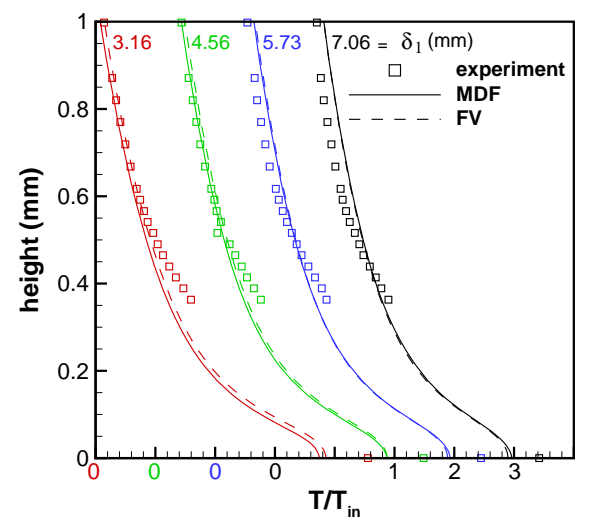

Figure 5: Normalized measured [54] and calculated (FV solver and hybrid MDF/FV scheme) temperature profiles at different displacement thicknesses in the near wall region of a turbulent Mach 4.5 boundary layer.

correctly and the wall temperatures of both simulations are basically identical.

No numerical problems occurred in this test case despite the high cell aspect ratio. The integration was very stable and CFL numbers of 3 and 0.4 are used for the FV and MDF solver, respectively. However, an implicit smoothing of the exchanged data according to Eq. (32) with $\varepsilon=0.1$ was required. The number of iterations needed to reach a steady-state solution is approximately 3 times higher for the MDF calculation compared to a pure FV simulation.

\subsection{Mach 1.2 flows with constant wall temperature}

The next two test cases treat flows with constant wall temperatures. The first one is a turbulent flow over a flat plate, the second one an axisymmetric flow through a weakly diverging pipe. Both test cases are purely academic, non-reacting, and without mixing. They are chosen to test the ability of the MDF solver to treat the enthalpy equation under these conditions correctly and to achieve identical results as a corresponding pure FV simulation. The latter one uses a transport equation for the total energy which includes kinetic and turbulent kinetic energy. For an agreement with the temperature profiles of the 

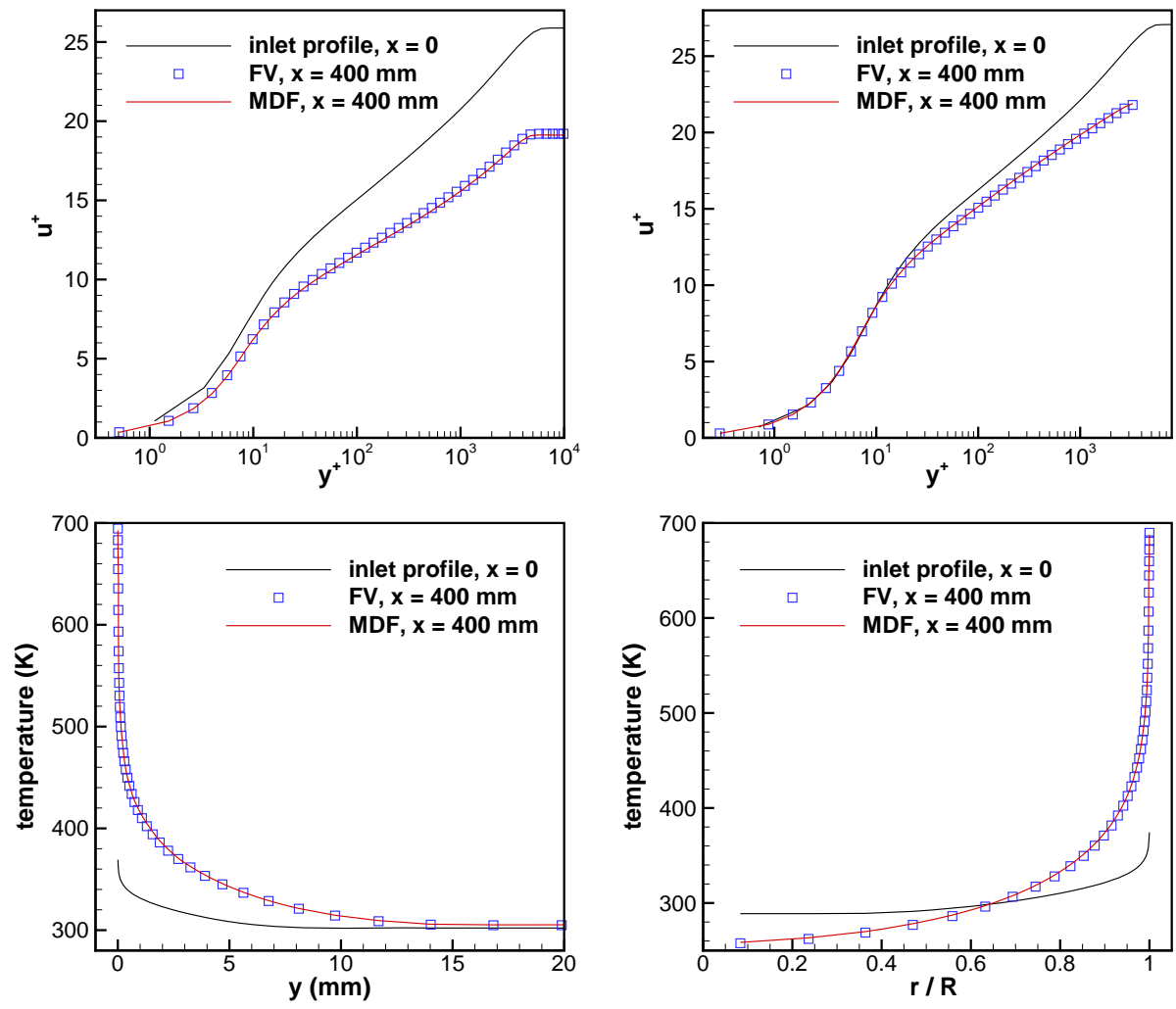

Figure 6: Comparison of calculated pure FV and hybrid MDF/FV results. Velocity (left side) and temperature (right side) at $x=400 \mathrm{~mm}$ for a turbulent non-reacting flow over a flat plate (left side) and through a pipe (right side).

particle solver the energy, momentum, and turbulent kinetic energy equations have to be implemented in a consistent way. Any particle in the MDF simulation which hits the wall takes the given wall temperature. In both cases (planar and axisymmetric) fully turbulent precalculated inlet profiles are chosen which are obtained using adiabatic wall boundary conditions. The inflow Mach number is 1.2 and the pressure is 1 bar. The pipe has a diameter of $R(x=0)=$ $29.4 \mathrm{~mm}$ at the inlet and the outward oriented angle is 0.29 degree. At the inlet the temperature boundary condition changes and for both simulations a constant wall temperature of $700 \mathrm{~K}$ is chosen. This causes a quick heating of the 
boundary layer due to the much warmer wall. Figure 6 shows velocity (upper figures) and temperature (lower figures) profiles for the flat plate (left side) and the pipe (right side) flow. Additionally, the precalculated inlet profiles for both cases are plotted. Results are given for the position $x=400 \mathrm{~mm}$. At the inlet ( $x=0 \mathrm{~mm}$ ) there is the sudden change in wall boundary condition. The results of the hybrid MDF/FV and the pure FV simulation are basically identical. This is required because the same modeling approaches are used, and therefore both simulation techniques fulfill the same first-order moment equations. The test cases demonstrate that heat conduction and viscous dissipation are modeled correctly on the particle level.

For both test cases no implicit smoothing of the transferred MDF data is required. CFL numbers of 6 and 0.4 are used for the FV and MDF solver, respectively. In the MDF simulations the averaged data strongly changed during the moving-time-averaging. Therefore the number of previous time-steps, over which the averaging is performed, had to be increased very slowly. Convergence of the pure FV solver is again much better due to a significantly higher CFL number (factor 3 to 4 ). In addition, an iteration of the Lagrangian particle solver is much more expensive than a FV step.

\subsection{Mach 2 turbulent flow over a backward facing step}

The last non-mixing, non-reacting test case is a turbulent Mach 2 flow over a backward facing step, which has been investigated experimentally by McDaniels et al. [56]. The channel has a length of $45 \mathrm{~mm}$, a step height of $3.18 \mathrm{~mm}$, and, after the step, a channel height of $21.29 \mathrm{~mm}$. The computational grid uses 2 blocks with $112 \cdot 80$ and $144 \cdot 112$ volumes, respectively. It is very fine in the near wall regions and achieves $y^{+}$-values below one. The cell aspect ratio is up to 850 near solid walls. Inlet profiles for the simulation have been calculated with the same code to match the experimentally measured boundary layer thickness of $\delta=1.45 \mathrm{~mm}$ at the $x=0$ location. The $\mathrm{AUSM}^{+}$-up [35] flux vector splitting is used together with a second or fourth order MLP discretization 

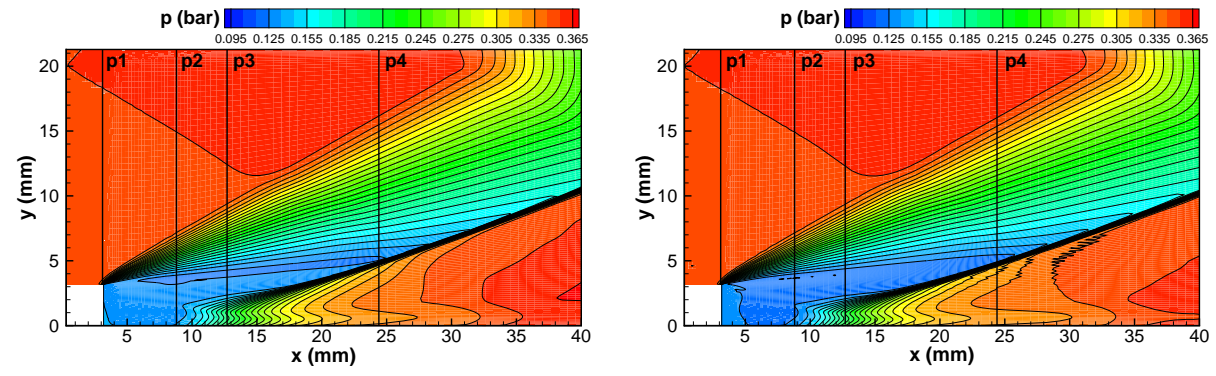

Figure 7: Comparison of calculated pressure distributions for a turbulent Mach 2 backward facing step flow. Left side pure FV simulation, right side hybrid MDF/FV simulation. The vertical lines indicate the positions ( $\mathrm{p} 1$ to $\mathrm{p} 4$ ) of profiles shown later.

$[34,29]$. However, for this steady-state test case, differences between second and higher order simulations are hardly visible [29]. As can be seen from Fig. 7, significant pressure differences occur, first in the expansion fan at the step and later due to the reattachment shock. Thus, the pressure derivative term in the enthalpy equation becomes important. Moreover, a large recirculation zone exists downstream of the step and there are significant temperature differences in the flow field. As can be seen from Fig. 7, the hybrid MDF/FV simulation (right side) agrees very well with the pure FV solution (left side). Downstream of the reattachment shock some slight oscillations are visible in the MDF results. Moreover, minor differences occur in the separated region and after the reattachment shock. The vertical lines p1 to p4 in Fig. 7 indicate positions, where experimental profiles are available $(x=3.1,8.8,12.7$, and 24.4 $\mathrm{mm}$ ). These profiles are given in Fig. 8. For the four positions pressure (upper) and temperature (lower) profiles are plotted. Given are results from the hybrid MDF/FV simulation and a pure FV calculation. The overall agreement between both simulations and the experimental data is very good. The velocity components (not shown) also agree very well. Minor differences occur only in the recirculation zone and in the region where the reattachment shock is formed. Even the increase in pressure and temperature over the reattachment shock is well reproduced by the MDF simulation. This is probably an advantage of a scalar thermochemical MDF because the positive shock capturing capabilities 

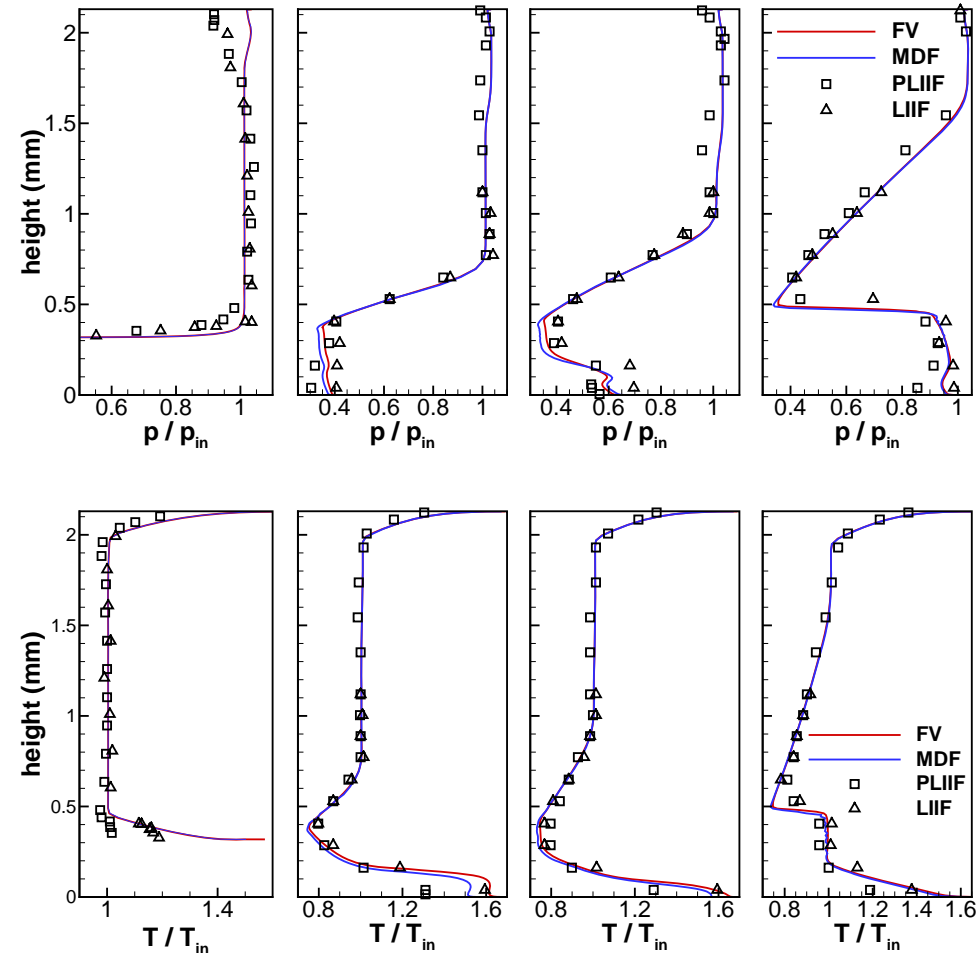

Figure 8: Comparison of hybrid MDF/FV results with those of a pure FV simulation and the experiment [56] for a turbulent Mach 2 backward facing step flow. Upper figures pressure, lower figures temperature profiles at the positions $\mathrm{p} 1$ to $\mathrm{p} 4$ (from left to right).

of the FV solver are maintained. Moreover, the first-order upwind character of the explicit particle solver does not introduce non-physical upwind influences at the shock wave. The simulation is numerically very stable in combination with approach 3 to model molecular transport. Using approach 1, the described problem of volumes without particles occurred as demonstrated in Fig. 3. Again, approximately 3 times more iterations are required for the hybrid MDF/FV scheme to obtain a converged solution compared to the standard FV method. 


\subsection{Subsonic Laminar Axisymmetric Non-Reacting Mixing Layer}

In RANS but also in LES simulations the solver should be able to treat laminar flows and differential diffusion. The following three test cases are chosen to demonstrate that

- the hybrid MDF/FV scheme works correctly in the limit of laminar flow (or at very low turbulence levels as in the next test case),

- modeling approach 3 not only stabilizes the numerical solution but also predicts differential diffusion very well.

Hence, a coaxial shear layer is simulated with $Y_{\mathrm{N}_{2}}=1$ in the outer and $Y_{\mathrm{O}_{2}}=$ $Y_{\mathrm{H}_{2}}=0.5$ in the inner flow. Both flows are separated for the first $50 \mathrm{~mm}$ (splitter plate) and mix over the next $150 \mathrm{~mm}$ downstream. The inner tube has a radius of $2 \mathrm{~mm}$, the outer one of $4 \mathrm{~mm}$. Thus, the splitter plate is located at $r=2 \mathrm{~mm}$. The inflow velocity, temperature, and pressure are $200 \mathrm{~m} / \mathrm{s}, 298 \mathrm{~K}$, and 1 bar, respectively. The flow is taken to be laminar to highlight the effect of differential diffusion. Figure 9 shows vertical profiles of the three species involved $137.5 \mathrm{~mm}$ downstream of the splitter plate. Given are profiles of the hybrid $\mathrm{MDF} / \mathrm{FV}$ simulation as well as pure FV results in an extension of the region where mixing takes place. The effect of differential diffusion is clearly visible due to the higher diffusivity of hydrogen compared to oxygen. Results of both simulation techniques agree very well. The profiles of the Lagrangian particle solver are a little bit more diffusive $\left(C_{m}=1\right)$ but the results show, that approach 3 fulfills all requirements given above.

Nevertheless, there are also limits associated with this technique as may be seen in Fig. 10. Here the influence of the model constant $C_{m}$ is investigated which is used in Eq. (64) to limit the rate of spurious variance reduction. The problem with a fast reduction is, that a short mixing time scale $\tau_{m}$ may cause a dissipative behavior of the solution in physical space, as described above. On the other hand, if $C_{m}$ is chosen too low, scalar mixing in thermochemical space is not fast enough and there may be non-physical variance. This has the effect 


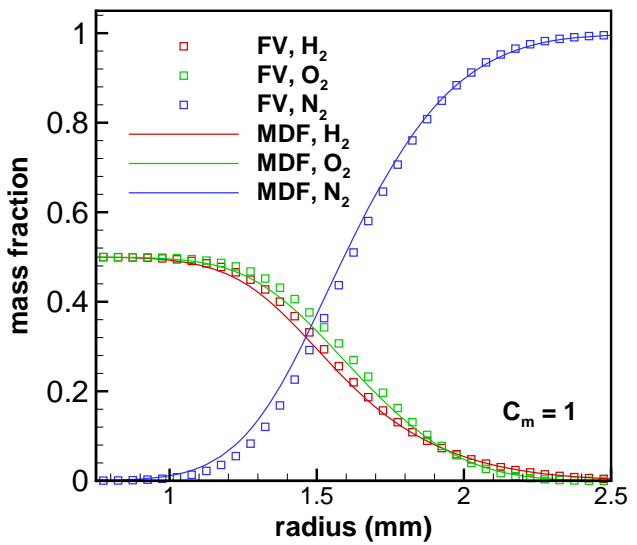

Figure 9: Comparison of pure FV and hybrid MDF/FV species profiles $137.5 \mathrm{~mm}$ downstream of the splitter plate for a laminar axisymmetric shear flow using $C_{m}=1$.

that on a particle level the species may be unmixed. In case of reactive flows this could prevent combustion. For this reason $C_{m}$ has to be chosen carefully. Note, however, this effect is important in laminar or weakly turbulent regions only. In fully turbulent flows the turbulent mixing time scale dominates $\left(\tau_{\Phi} \ll \tau_{m}\right)$ and it follows from Eq. (59) that $\tau_{g} \approx \tau_{\Phi}$. In case of the fully turbulent backward facing step flow from Sect. 6.3 a change of $C_{m}$ from one to five had no visible effect at all. In Fig. 10, species profiles are plotted on the left side at $x=137.5 \mathrm{~mm}$ downstream of the splitter plate. On the right side the difference between the $\mathrm{O}_{2}$ and $\mathrm{H}_{2}$ mass fractions is given to highlight the effect of differential diffusion and isolate it from the remaining diffusion process. The species profiles (left side) show that the solution becomes more diffusive if $C_{m}$ is increased. The same tendency is observed if the order of discretization in the pure FV scheme is reduced from second to first order. The best agreement with the FV solution is obtained with the smallest value $C_{m}=0.25$. Nevertheless, the MDF solution using $C_{m}=1$ still agrees pretty well with the second-order FV results. The behavior of $Y_{\mathrm{O}_{2}}-Y_{\mathrm{H}_{2}}$ (right side) is more complex. No chosen value of $C_{m}$ is able to correctly reproduce the FV result. There are two points which 

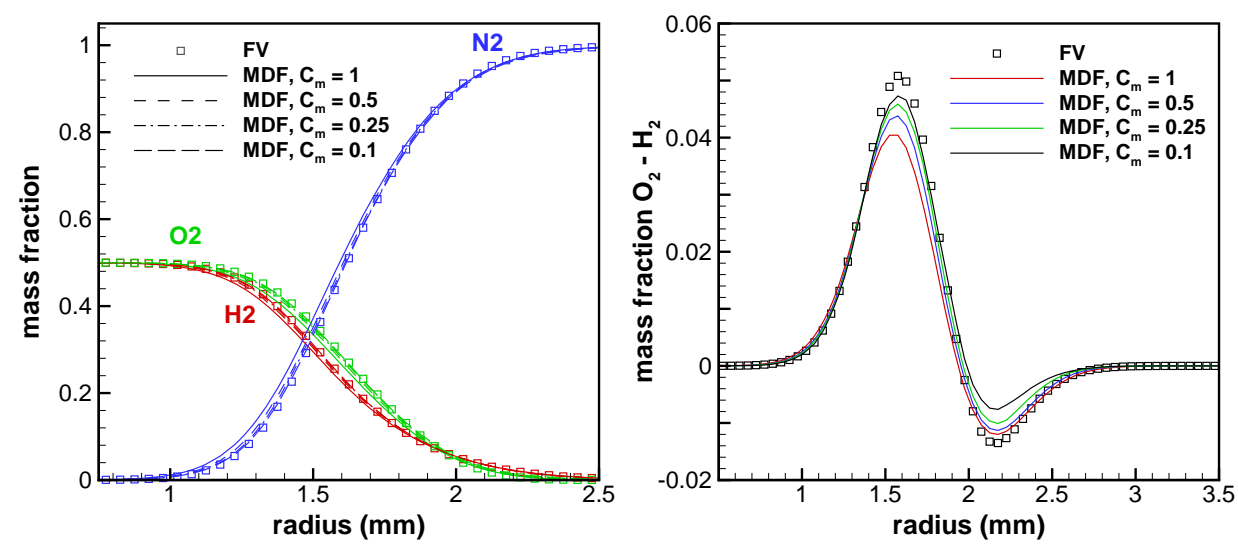

Figure 10: Comparison of pure FV and hybrid MDF/FV species profiles (left side) and difference between $\mathrm{O}_{2}$ and $\mathrm{H}_{2}$ mass fractions (right side) for a laminar axisymmetric shear flow and different values of $C_{m}$. All profiles are $137.5 \mathrm{~mm}$ downstream of the splitter plate.

may be responsible for these deviations: The numerical diffusion caused by $\tau_{m}$ in the IEM model which scales with the parameter $C_{m}$ and the fact, that one free parameter $\left(\tau_{m}\right)$ is available for a multi-scalar problem only. Fig. 10 (right side) also shows, that the recommended value $C_{m}=1$ is a good compromise. Despite the discrepancies the new technique offers a high potential for complex flow simulations. Moreover, the height where differential diffusion takes place is predicted very well.

In order to highlight the spurious variance production a parameter is needed which evaluates the degree of mixing achieved on the particle level. For this purpose the following parameter is chosen

$$
\nu_{\text {mix }}=\frac{\widetilde{Y_{A} Y_{B}}}{\widetilde{Y}_{A} \widetilde{Y}_{B}}=1+\frac{\widetilde{Y_{A}^{\prime \prime} Y_{B}^{\prime \prime}}}{\widetilde{Y}_{A} \widetilde{Y}_{B}}
$$

which should be 1 in case of perfect mixing (laminar flow) and otherwise is a measure for the non-physical covariance between $A$ and $B$. Here $\nu_{m i x}$ is calculated with $A=\mathrm{O}_{2}$ and $B=\mathrm{N}_{2}$ from the particle properties. Figure 11 shows on the left side the degree of mixing at $x=137.5 \mathrm{~mm}$ using different 

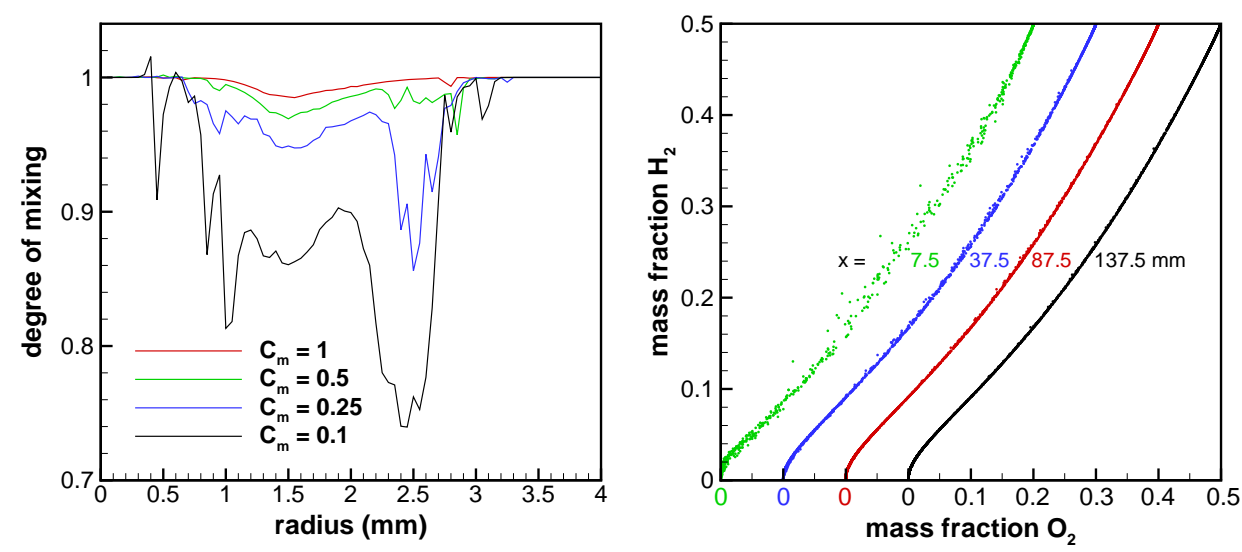

Figure 11: Influence of the modeling parameter $C_{m}$ for a laminar axisymmetric shear flow. Left side degree of mixing (normalized species covariance) at $x=137.5 \mathrm{~mm}$, right side $\mathrm{H}_{2}-\mathrm{O}_{2}$ scatter plot of particle properties along a vertical line at four $x$-positions.

values $C_{m}$. In order to reduce the statistical error, the particle data is averaged over 1000 iterations at steady-state conditions. While high values for $C_{m}$ may be negative due to the numerical diffusion of the IEM model, its effect on $\nu_{m i x}$ is in the opposite direction. A higher value of $C_{m}$ causes a faster mixing and a better agreement with the laminar value of one. As can be seen from Fig. 11 (left side), the spurious covariance is nearly completely removed in case of $C_{m}=1$. On the right side of Fig. 11 scatter plots of particle properties $\left(\mathrm{H}_{2}\right.$ over $\mathrm{O}_{2}$ mass fractions) are shown for all particles on a vertical line at a given axial position. The four scatter plots are for $x=7.5,37.5,87.5$, and 137.5 $\mathrm{mm}$ downstream of the splitter plate. These results are obtained with $C_{m}=$ 1. Note, that for a better representation, the $x$-origins in this figure are shifted for the different scatter plots. It can be seen that with the exception of the plot for $x=7.5 \mathrm{~mm}$ all points basically collapse into a line. Even for $x \leq 37.5$ $\mathrm{mm}$ the scattering is relatively low. Both plots of Fig. 11 demonstrate, that $C_{m}=1$ is a good compromise between a fast mixing (low spurious variance production) and a good spatial resolution. The achieved results are comparable to the second-order FV profiles. 
The variance in the species distribution also affects the smoothness of the data transferred to the FV scheme and thus numerical stability. In case of $C_{m}=1$ or higher, no implicit smoothing of the exchanged data is required. For $C_{m}=0.5$ and lower, however, an implicit smoothing was necessary in the transient phases of the simulation. After reaching a statistical steady-state and with the start of the moving-time-averaging, the implicit smoothing could be switched off.

\subsection{Subsonic Weakly Turbulent Axisymmetric Non-Reacting Mixing Layer}

The test case from the last subsection is repeated here with the difference that the inflow in not laminar but at a low turbulence level (the $q-\omega$ turbulence model [30] is used). The turbulence intensity is $0.816 \%$ in the inner $\mathrm{H}_{2} / \mathrm{O}_{2}$ flow and $0.408 \%$ in the outer $\mathrm{N}_{2}$ flow. In this way the eddy viscosity at the inlet is of the same order as the molecular viscosity (factor 1.38 and 2.35). The turbulence level significantly increases in the shear layer where the impact of molecular diffusivity is disappearing. Due to the dominance of turbulent mixing (IEM model), the effect of $C_{m}$ is also reduced. Figure 12 shows on the left side species profiles $137.5 \mathrm{~mm}$ downstream of the splitter plate. As expected, the much stronger turbulent mixing and diffusion causes much more homogeneous profiles and the impact of differential diffusion is negligible (the profiles of $\mathrm{H}_{2}$ and $\mathrm{O}_{2}$ become identical). Besides the MDF/FV simulation with approach 3 for molecular transport and scalar mixing an additional calculation is performed with the standard IEM model using $1 / \tau_{m}=0$. This is done to highlight the non-physical variance production of approach 3. As can be seen from Fig. 12 (left side) the use of $\tau_{m}$ does not change the mean species profiles. On the right-hand side of Fig. 12 the impact of $\tau_{m}$ on the degree of mixing between $\mathrm{O}_{2}$ and $\mathrm{N}_{2}$ is investigated. Plotted are profiles at 4 streamwise positions. Directly downstream of the splitter plate $(x=7.5 \mathrm{~mm})$, where the flow still has a low turbulence level, the simulations with approach $3\left(C_{m}=1\right)$ and with the standard IEM model show small differences. They disappear further downstream 

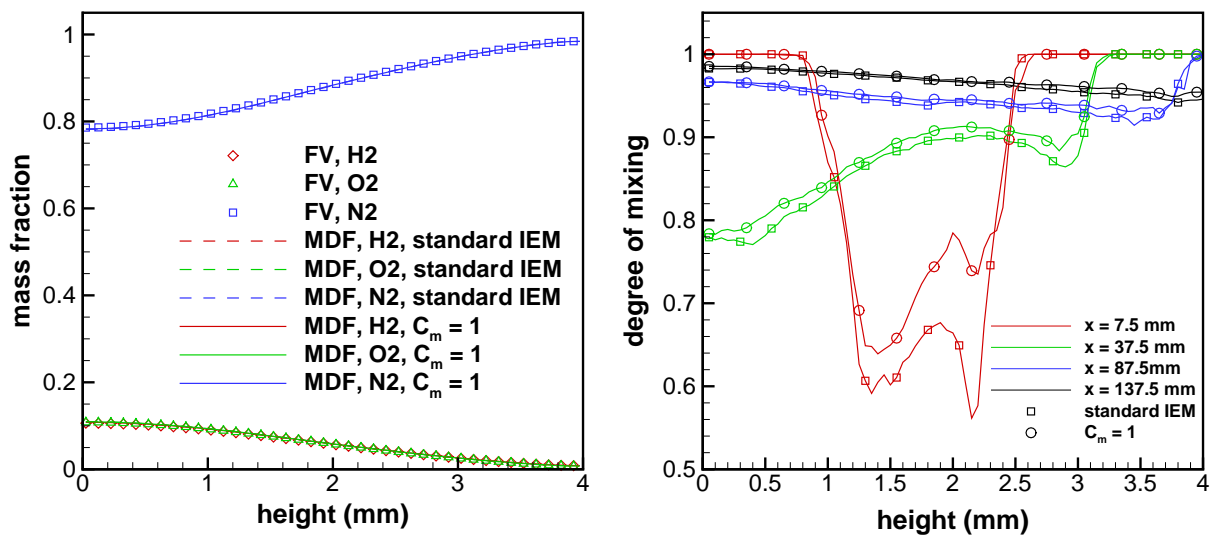

Figure 12: Comparison of pure FV and hybrid MDF/FV species profiles at $x=137.5 \mathrm{~mm}$ (left) and degree of mixing at different streamwise positions (right). Test case is a weakly turbulent axisymmetric non-reactive shear flow.

where both profiles become more and more identical due to the dominance of turbulent mixing and diffusion.

\subsection{Laminar Supersonic Hydrogen Flame}

As shown in the last two non-reactive test cases, the unwanted non-physical variance induced by approach 3 can be reduced successfully if the new mixing time scale $\tau_{g}$ (see Eq. (59)) is used in the IEM model. Moreover, the model constant $C_{m}=1$ offers a good compromise between accuracy and numerical diffusion. The next check is how this model performs when applied to reactive flows. As described in Sect. 5.3.1, even at local equilibrium conditions there may be a strong impact from combustion on the species variances and covariances to deviate from the approximation given in Eq. (81). Thus it is expected that the single reduction time scale $\tau_{m}$ is not able to completely compensate for the spurious production terms.

Again a laminar academic flame is chosen to highlight the effects of $\tau_{m}$ and $C_{m}$. The setup is based on a planar supersonic flow over a splitter plate. 

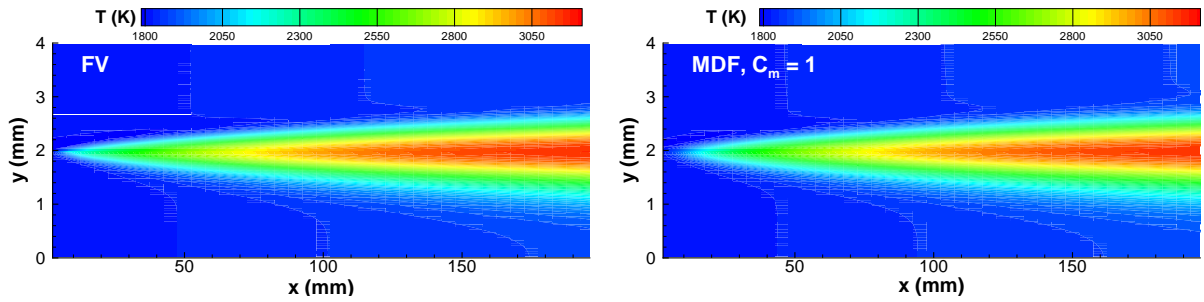

Figure 13: Comparison of calculated temperature distributions for a supersonic hydrogen flame. Left side FV simulation, right side hybrid MDF/FV results using $C_{m}=1$.

The lower flow is a hydrogen/nitrogen mixture $\left(Y_{\mathrm{H}_{2}}=0.25, Y_{\mathrm{N}_{2}}=0.75\right)$ and the upper flow is pure oxygen $\left(Y_{\mathrm{O}_{2}}=1\right)$. The inflow velocities, temperatures, and pressures of both streams are $3000 \mathrm{~m} / \mathrm{s}, 1800 \mathrm{~K}$, and 1 bar, respectively. A reduced 7-species, 7-step reaction mechanism [57] is employed for hydrogen combustion. Due to the high inflow temperatures chemistry is very fast and close to equilibrium conditions. Figure 13 shows temperature contours of a pure FV (left side) and a hybrid MDF/FV simulation (right side). The latter one uses the constant $C_{m}=1$. Both figures are nearly identical. Minor differences occur at $x \approx 0 \mathrm{~mm}$ (end of the splitter plate) due to large species gradients between the upper and lower streams. The mixing limitation with $C_{m}=1$ is slightly too strong at this position, but on the other hand avoids a smearing of the species profiles due to numerical diffusion. This is shown in more detail on the left side of Fig. 14. Given are results from a pure FV simulation and hybrid $\mathrm{MDF} / \mathrm{FV}$ results with $C_{m}=1,2,3$, and 5 for the positions $x=48$ and 198 $\mathrm{mm}$ downstream of the splitter plate. The profiles at $48 \mathrm{~mm}$ clearly show that the maximum temperature for the $C_{m}=1$ simulation is slightly lower than the FV result. An accelerated mixing $C_{m} \geq 2$ achieves a perfect matching with the FV maximum temperature but at the wings of the profile the solutions become too diffusive. At $x=198 \mathrm{~mm}$ the differences in maximum temperature have disappeared, but the dissipative character of the solutions with $C_{m}>1$ becomes even more visible. It is concluded that a value of $C_{m}=1$ is the best choice. On the right-hand side of Fig. 14 temperature profiles using $C_{m}=1$ are plotted for 

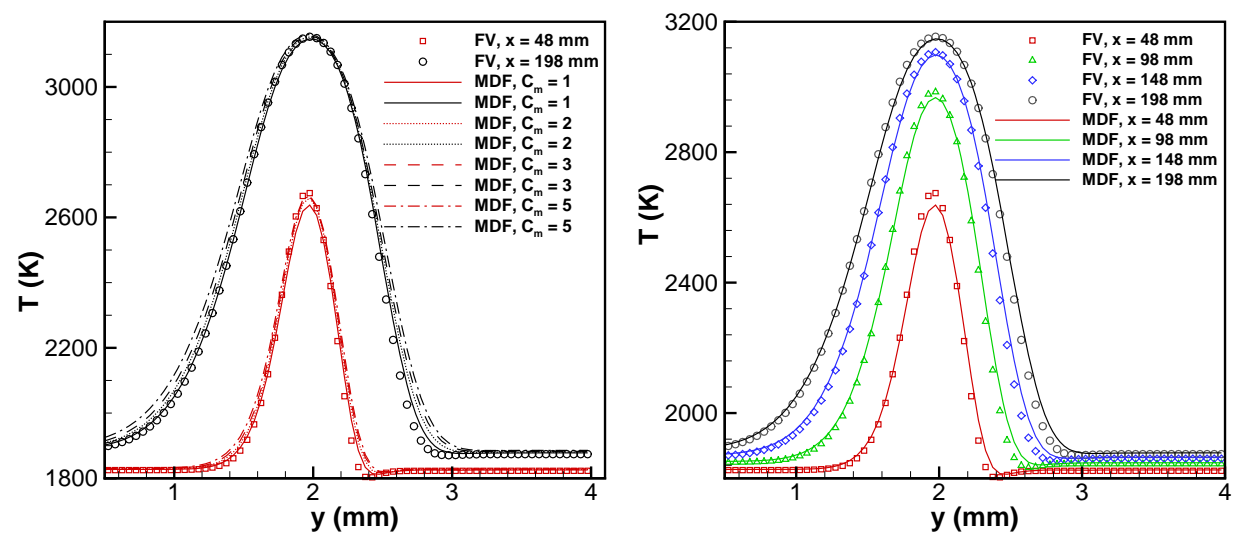

Figure 14: Comparison of pure FV and hybrid MDF/FV temperature profiles at 2 spatial position for different values of $C_{m}$ (left) and temperature profiles for $C_{m}=1$ at different $x$-positions (right). Test case is a laminar supersonic hydrogen flame.

different positions downstream of the splitter plate. With the exception of the first profile the overall agreement between the particle and the FV solver is very good. This can also be stated for the mean species profiles, which are given in Fig. 15 for $x=148 \mathrm{~mm}$. Even the radicals predicted by the hybrid FV/MDF scheme agree very well with those of the pure FV solution.

Due to the very high inflow temperatures of $1800 \mathrm{~K}$, chemistry is extremely fast. This can be clearly seen in the composition scatter plots for $\mathrm{O}_{2}$ and $\mathrm{H}_{2}$ which are shown for $x=48,98,148$, and $198 \mathrm{~mm}$ in Fig. 16. The properties from all particles in volumes along vertical lines at the given $x$-positions are extracted for this plot. For all $x$-positions the properties basically collapse into one line, indicating fast chemistry.

Next the occurrence of non-physical variance is investigated. In addition to the averages, the rms (root mean square) values of species fluctuations of the hybrid FV/MDF simulation are plotted in Fig. 15 for the position $x=198 \mathrm{~mm}$. These values are calculated by averaging the particle data over 1000 iterations at steady-state conditions. Ideally all variances should be zero for this laminar combustion test case. However, spurious variances occur for all species in the 

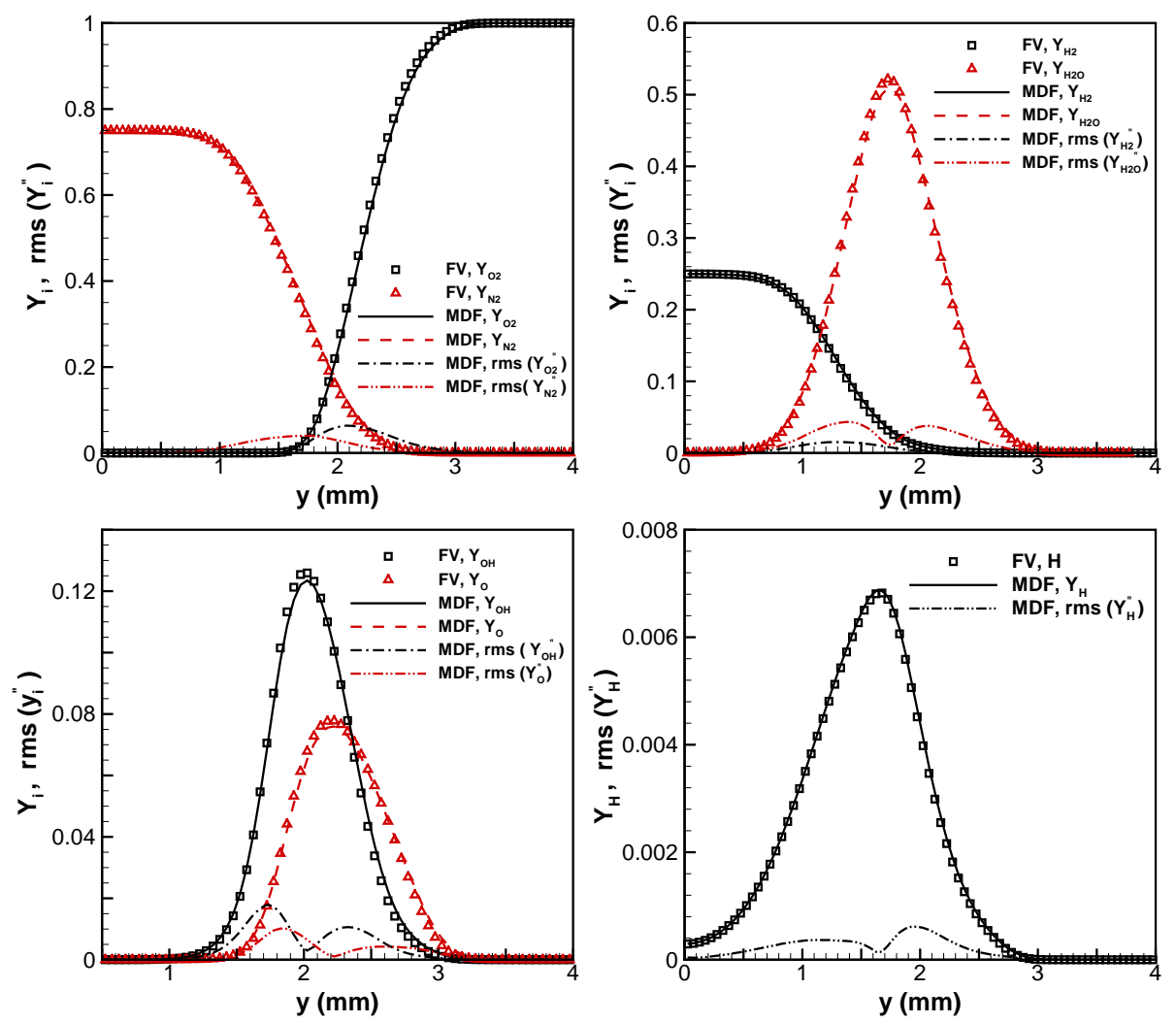

Figure 15: Comparison of pure FV and hybrid MDF/FV mean species and rms species fluctuation profiles at $x=198 \mathrm{~mm}$ downstream of the splitter plate using $C_{m}=1$. Test case is a laminar supersonic hydrogen fiffusion flame (upper figures: $\mathrm{O}_{2}$ and $\mathrm{N}_{2}$ left, $\mathrm{H}_{2}$ and $\mathrm{H}_{2} \mathrm{O}$ right, lower figures: $\mathrm{OH}$ and $\mathrm{O}$ left, $\mathrm{H}$ right).

main reaction zone. These variances are significantly higher than in the nonreactive mixing test case from Sect. 6.4 (see the line for $C_{m}=1$ on the left side of Fig. 11). If $C_{m}=5$ is taken instead of $C_{m}=1$, the spurious variances are reduced by nearly $50 \%$. This, however, increases the numerical diffusion of the IEM model as shown before in Fig. 14. The spurious variances are a consequence from modeling molecular diffusion by the Wiener process. On the other hand, this measure is required to ensure numerical stability. Moreover, the spurious variance production still is relatively low and the mean species profiles are in a very good agreement with the FV results. In addition, this 


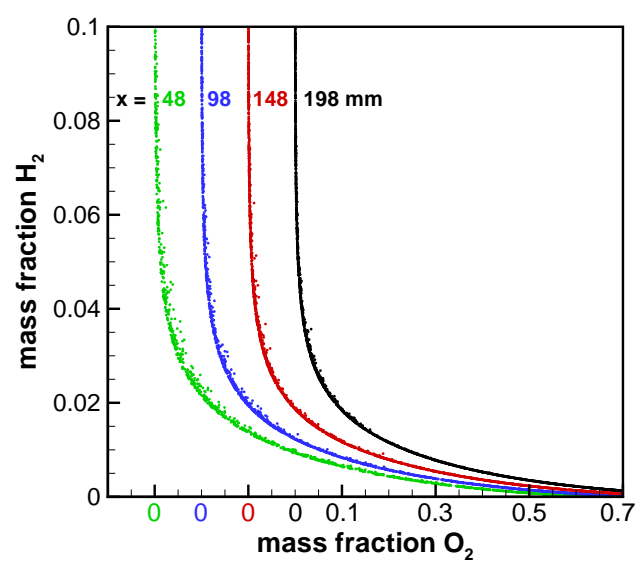

Figure 16: Composition scatter plots of $\mathrm{O}_{2}$ and $\mathrm{H}_{2}$ at different $x$-positions for a laminar supersonic hydrogen flame ( $x$-origin is shifted from plot to plot).

effect become important in regions of disappearing turbulence only.

No implicit smoothing of the transferred data (from MDF to the FV scheme) was required for this combustion test case. However, due to the lower CFL number ( 0.4 compared to 3 for the FV solution) the number of iterations needed to achieve a steady-state solution is 6 times higher for the hybrid MDF/FV scheme.

Based on the results of the previous test cases approach 3 can be used for practical high speed applications. Without the described technique to treat the molecular transport as part of the Wiener process, it was impossible to simulate the experiments from next Sects. 6.7 and 6.8.

\subsection{Supersonic Mixing Channel}

Finally, two practical test cases are presented. The first one is related to supersonic combustion and treats a Mach 2 non-reacting supersonic mixing channel with hydrogen supply by a strut injector. The turbulent flow simulation starts at the air nozzle throat (required in the experiment) with sonic condi- 

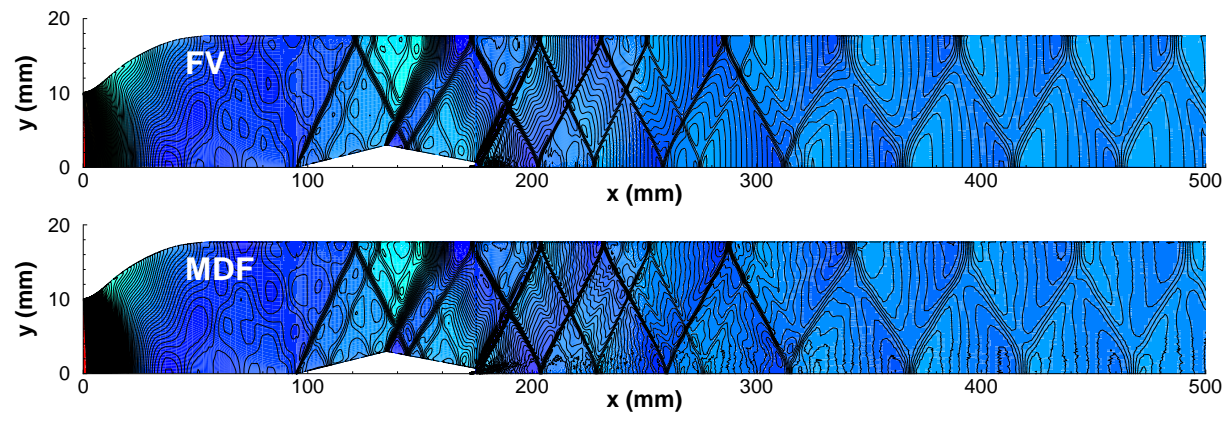

Figure 17: Pressure contours for simulations of a supersonic mixing channel with hydrogen strut injection. Upper figure pure FV simulation, lower figure hybrid MDF/FV solution.

tions and $240 \mathrm{~K}$ and 2.05 bar for temperature and pressure, respectively [58]. Inside the blunt end of the strut is a nozzle through which hydrogen is injected. The nozzle is resolved by the computational grid. The sonic hydrogen injection conditions are $260 \mathrm{~K}$ and 1.95 bar for temperature and pressure, respectively [58]. In this case the thermochemical MDF covers the enthalpy and the mass fractions of oxygen, hydrogen, and nitrogen. The computational grid consists of 3 blocks (which later are divided for the parallel simulation) with $472 \cdot 33$, $442 \cdot 56$, and $672 \cdot 96$ volumes, respectively. The grid is strongly refined in the mixing zone and close to solid walls. Figure 17 shows calculated pressure contours using the fifth order MLP discretization [29] in both FV parts. In the upper figure results of a pure FV simulation are given, in the lower one from the hybrid MDF/FV calculation. To our knowledge this is the first simulation where such a multiple shock reflection test case is simulated by a hybrid $\mathrm{FV} /$ Lagrangian particle method. In case of the MDF simulation the pressure contours are somewhat noisy downstream of the strut. The implicit smoothing of transferred variables was essential in this test case $(\varepsilon=0.1)$. Moreover, a moving-time-averaging of transferred MDF data was possible over a relatively small number of previous time-steps $(\leq 40)$ only. Otherwise artificial shock systems are induced. However, the shock resolution of the MDF simulation is very good and better than expected for a test case with strong pressure and temperature gradients. 


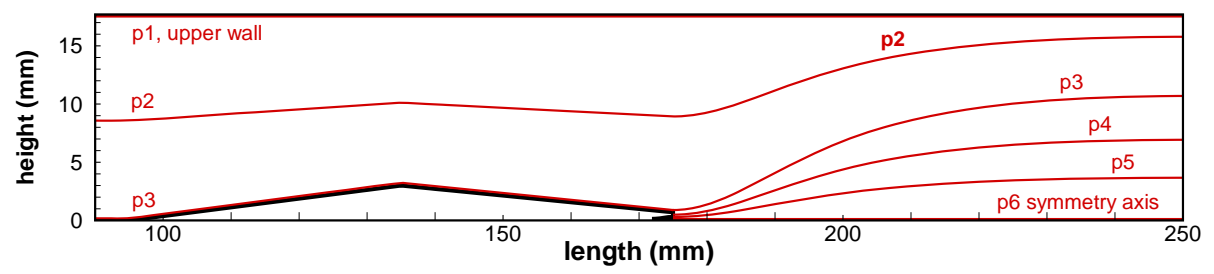

Figure 18: Extension of the channel geometry around the strut injector. The lines p1 to p6 indicate the positions of profiles plotted in Fig. 19.

For a more detailed analysis, pressure and temperature profiles are compared. The locations of the 6 profiles $\mathrm{p} 1$ to $\mathrm{p} 6$ investigated (along the curvilinear grid) are shown in Fig. 18 in an extension of the region around the strut injector. Profile $\mathrm{p} 1$ is along the upper wall, $\mathrm{p} 2$ is in the first part in the middle of the channel, $\mathrm{p} 3$ is in the first part along the symmetry axis and on the strut surface, $\mathrm{p} 6$ is along the symmetry axis in the rear part, and $\mathrm{p} 4$ and $\mathrm{p} 5$ are in between. Figure 19 shows the six pressure (left side) and temperature (right side) profiles obtained from the pure FV and hybrid MDF/FV simulation. Along the channel wall experimentally measured pressures are available which are given in the pressure plot, too. The pressure profiles demonstrate, that the strong pressure differences due to expansion in the nozzle and the pressure changes over the shock waves are well reproduced in the MDF solution. The differences compared to the high order pure FV solution are marginal. This is in contrast to the temperature profiles, which, at least further downstream, show some minor deficiencies compared to the FV results. Nevertheless, the agreement is very good. The results show that the pressure derivatives in the enthalpy equation as well as viscous transport and dissipation in the boundary layer are correctly predicted by the Lagrangian particle method. No negative influence is observed from the lower spatial order of the particle solver, what probably is an advantage of using a thermochemical MDF. Thus, all requirements are fulfilled to use the thermochemical MDF for compressible combustion in high speed flows. 

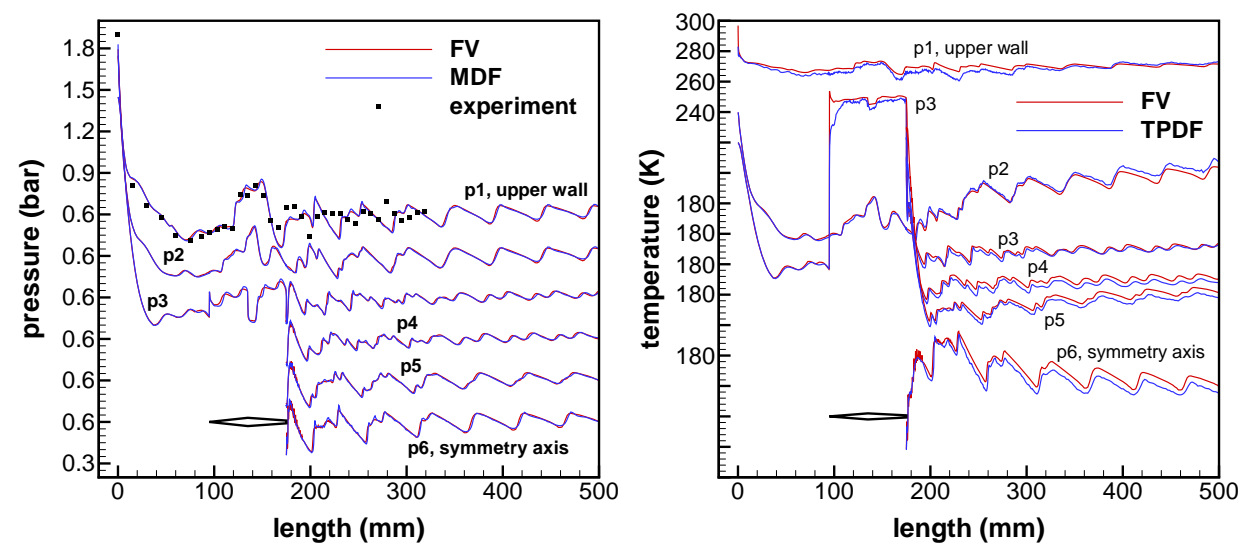

Figure 19: Comparison of pure FV and hybrid MDF/FV pressure (left) and temperature (right) profiles for a supersonic mixing channel. The position of the strut is also given in both figures (the $y$-origins are shifted for the different profiles) .

\subsection{PennState Preburner Combustor}

The final test case is a model rocket combustor which has been investigated experimentally at the Pennsylvania State University [59]. The experiment has been designed to characterize the wall heat transfer, which is an important issue in rocket combustor design. The combustor is axisymmetric and has a diameter of $38.1 \mathrm{~mm}$ and a length of $286 \mathrm{~mm}$. Two upstream preburners produce oxidizerrich and fuel-rich gases, respectively. The combustor is operated at a pressure of 5.42 MPa. Details concerning geometry and operating conditions may be found in Ref. [59]. Steady-state RANS and hybrid MDF/FV simulations are performed for this test case. It is known [60], that there are unsteady effects in the flow field of this combustor and that a LES or DDES should be preferred. However, the emphasis of this paper is not on an accurate simulation of the test case but on the demonstration of the ability of the hybrid solver to deal with such problems. For hydrogen/oxygen combustion a reduced 7-species, 7-step reaction mechanism is used [57] and for turbulence closure the $q-\omega$ low-Reynolds number turbulence model [30]. In the experiment combustor wall temperatures have been measured. These temperatures are used in a least square fit to obtain the 


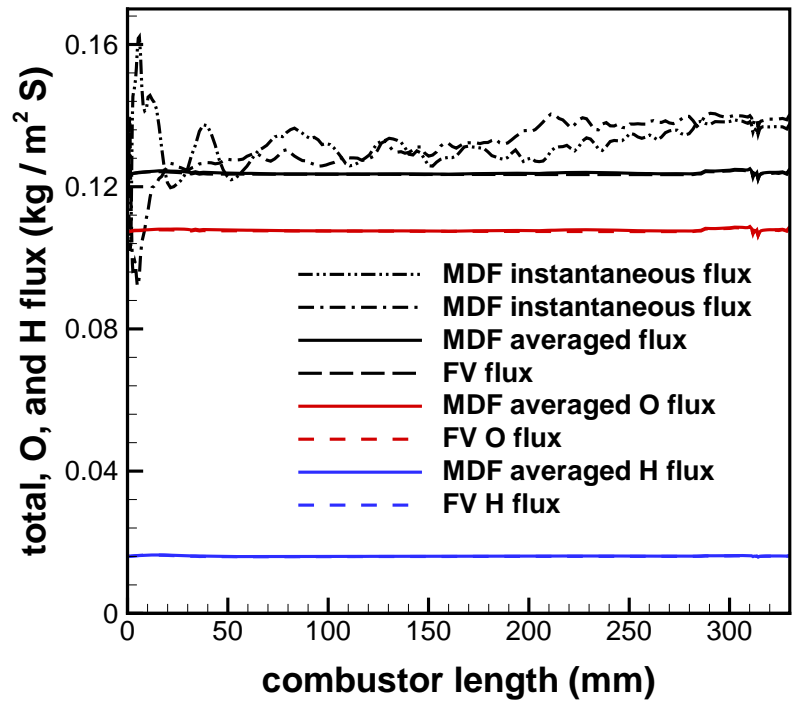

Figure 20: Calculated total and element ( $\mathrm{O}$ and $\mathrm{H})$ mass fluxes along the combustor length. Given are instantaneous and averaged MDF/FV results as well as pure FV values for simulations of the PennState model rocket combustor).

wall temperatures for the numerical simulation. The computational grid has about 50,000 volumes and is strongly refined near the oxidizer post tip and at all near wall regions. A constant mass flow boundary condition is chosen for both inflows.

Strong oscillations in mass flow but also in other variables occurred during the transient phases of the hybrid MDF/FV simulation (before the moving-timeaveraging is started). This caused stability problems for the solver and required a much stronger implicit smoothing of the transferred MDF data $(\varepsilon=0.6)$ than before. Moreover, similar time-steps had to be chosen in the FV and particle solvers. Thus the CFL number of 0.4 is maintained in the particle part, while the CFL number in the FV step is reduced to 1 . Figure 20 shows cross section averaged mass fluxes along the combustor length which, at steady-state conditions, should be constant. Besides the total mass flux the calculated element 

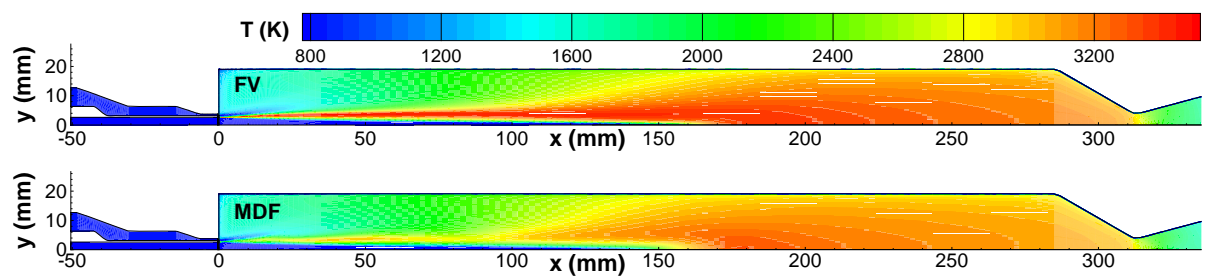

Figure 21: Calculated temperature contours of the PennState model rocket combustor. Upper figure pure FV simulation, lower figure hybrid MDF/FV calculation.

mass fluxes of $\mathrm{O}$ and $\mathrm{H}$ (calculated from the different species mass fractions) are plotted. For the total mass flux two instantaneous profiles are given at arbitrarily chosen times shortly before the moving-time averaging is started. They show strong fluctuations of this already cross section averaged value. Close to the injector at $x=0 \mathrm{~mm}$, the total mass flux fluctuates by more than $25 \%$ in positive and negative direction. With increasing combustor length the instantaneous mass flux is more and more overpredicted. Reaching the combustor nozzle at $x \approx 315 \mathrm{~mm}$, both instantaneous mass fluxes are more than $10 \%$ too high. The strong fluctuations of the instantaneous flow field are induced by the particle solver and do not appear in the pure FV simulation. After a statistically steady-state solution is obtained, the MDF data is averaged over 2000 previous time-steps. This stabilizes the simulation and causes averaged mass fluxes for the $\mathrm{MDF} / \mathrm{FV}$ simulation which are almost identical to the pure FV results (see Fig. 20). However, in contrast to some of the previous test cases, the strong spatial smoothing of the transferred MDF data could not be switched off after the averaging process is started.

In the Figs. 21 and 22 temperature and $\mathrm{OH}$ mass fraction contours are given for both types of simulation, respectively. Each upper figure shows results from a pure FV simulation, the lower figures are from the hybrid MDF/FV scheme. Significant differences are obtained between both simulations which are purely attributed to the treatment of the chemical source term. In the hybrid MDF/FV solution both temperature and $\mathrm{OH}$ are more diffuse with lower maximum values. This differing flame behavior affects the wall heat flux, too. Figure 23 shows 

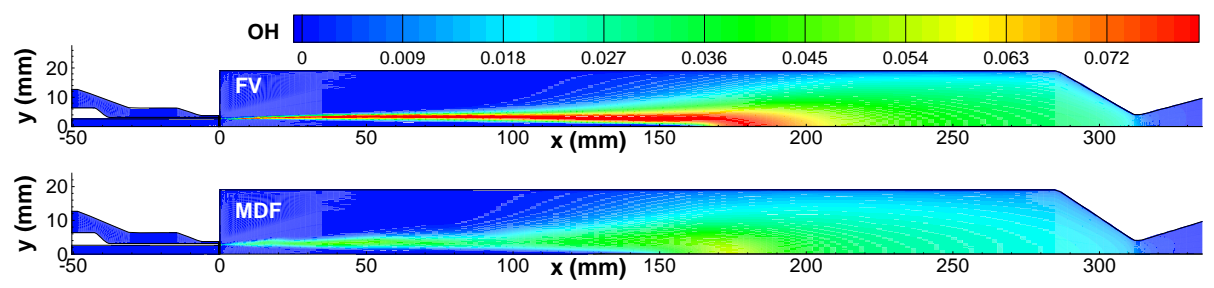

Figure 22: Calculated $\mathrm{OH}$ mass fraction contours of the PennState model rocket combustor. Upper figure pure FV simulation, lower figure hybrid MDF/FV calculation.

calculated and measured wall heat fluxes along the combustor length. Even if the differences are not very strong, the $\mathrm{MDF} / \mathrm{FV}$ solution represents the experimental tendencies better than the pure FV results.

Finally, the thermochemical particle data is investigated. Figure 24 shows scatter plots of particle temperatures over and the mass fractions of $\mathrm{OH}$ (upper figures) and $\mathrm{O}$ (lower figures). These data is obtained on vertical lines through the combustor. Both plotted results are on lines close to the oxygen and hydrogen supply tubes, the first one $0.5 \mathrm{~mm}$, the second one $2 \mathrm{~mm}$ downstream of the injector. Symbols indicate different regions along these lines (squares are from the region of the oxygen jet close to the symmetry axis, circles are downstream of the injector post which is the next block in radial direction). A strong scattering occurs at these positions. This is despite the high pressure of 54 bar and the resulting fast chemistry and demonstrates, that simple combustion models can fail in such regions. To our knowledge this is the first Lagrangian particle MDF solution with finite-rate chemistry of a model rocket combustor.

\section{Conclusions}

A thermochemical MDF/FV approach for compressible high speed flows (RANS) is presented. Due to the high cell aspect ratios required to resolve the viscous sublayer, stability problems for the particle solver arise. It was found, that the treatment of molecular transport by the stochastic Wiener process 


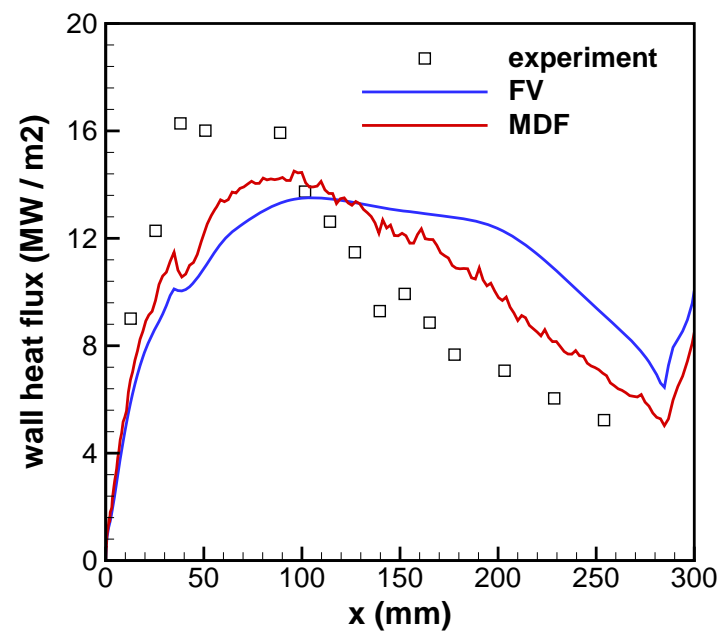

Figure 23: Calculated wall heat fluxes of a pure FV and a hybrid MDF/FV simulation and experimental values [59] for the PennState model rocket combustor.

stabilizes the simulation even in complex cases. Based on this result a new model for differential diffusion is developed. The new model can be used in LES, too. In a detailed analysis it is shown, that for the required modeling constant a good compromise can be achieved between numerical dissipation and non-physical variance. In a number of test cases it is demonstrated, that

- terms only appearing in compressible flows (e.g. pressure derivatives, viscous work) are implemented consistently to the FV solver,

- high speed boundary layers with adiabatic and isothermal walls are well predicted,

- complex shock systems can be simulated with particle methods and that an excellent shock resolution is achieved,

- all simulations are relatively stable, what is important for practical applications. However, an implicit smoothing of transferred particle data was required in some cases. 

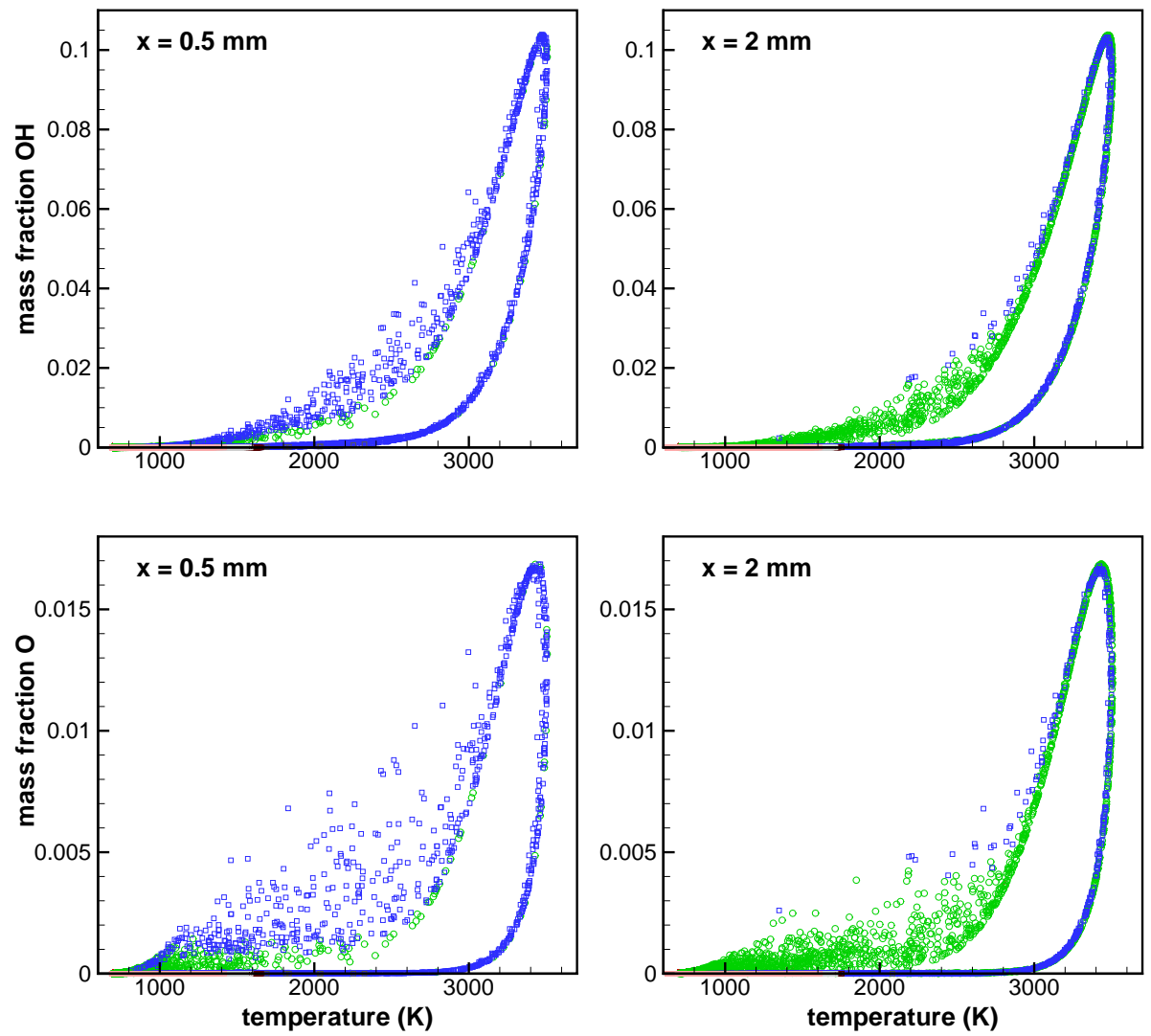

Figure 24: Scatter plots of MDF particle data along vertical lines at $x=0.5$ and $x=2 \mathrm{~mm}$ downstream of the injector. Test case is the PennState model rocket combustor

Moreover, the first simulations of a supersonic mixing channel with multiple shock reflections and of a model rocket combustor with finite-rate chemistry are presented. Results from the latter case show, that MDF methods are important in rocket combustion because the described features are neglected in less sophisticated combustion models. 


\section{Acknowledgements}

Parts of this work have been performed within the framework of the research program SFB-TR 40, funded by the German Research Foundation (DFG). The author wishes to thank the DFG for financial support. Moreover, the author also thanks the DLR (German Aerospace Center) for support within the DLR project ProTau. Most simulations have been performed at the High Performance Computing Center Stuttgart (HLRS). Finally, the author thanks Dr. Andreas Fiolitakis for numerous discussions on the topic.

\section{References}

[1] S. B. Pope, A Monte Carlo method for the PDF equations of turbulent reactive flows, Combustion Science and Technology 25 (1981) 159-174.

[2] S. B. Pope, PDF methods in reactive flows, Progress in Energy and Combustion Science 11 (1985) 119-192.

[3] S. B. Pope, Turbulent premixed flames, Annual Review of Fluid Mechanics 19 (1987) 237-270.

[4] S. B. Pope, S. M. Correa, Joint PDF calculations of a non-equilibrium turbulent diffusion flames, in: Proceedings of the Combustion Institute, Vol. 21, 1988, pp. 1341-1348.

[5] A. T. Hsu, A study of hydrogen diffusion flames using pdf turbulence model, AIAA paper 91-1780 (1991).

[6] Y. Z. Zhang, D. C. Haworth, A general mass consistency algorithm for hybrid particle/finite-volume PDF methods, Journal of Computational Physics 46 (2003) 156-193.

[7] S. James, J. Zhu, M. S. Anand, Lagrangian PDF transport methods for simulations of axisymmetric turbulent reacting flows, AIAA paper 20050156 (2005). 
[8] R. McDermott, S. B. Pope, A particle formulation for treating differential diffusion in filtered density function methods, Journal of Computational Physics 226 (2011) 947-993.

[9] S. Viswanathan, H. Wang, S. B. Pope, Numerical implementation of mixing and molecular transport in LES/PDF studies of turbulent reacting flows, Journal of Computational Physics 230 (2011) 6916-6957.

[10] A. Ansari, P. H. Pisciuneri, P. A. Strakey, P. Givi, Scalar-filtered massdensity-function simulation of swirling reacting flows on unstructured grids, AIAA Journal 50 (11) (2012) 2476-2482.

[11] A. Banaeizadeh, A. Afshari, F. Jaberi, Large-eddy simulation of turbulent flows in internal combustion engines, International Journal of Heat and Mass Transfer 60 (2013) 781-796.

[12] D. C. Haworth, S. H. El Tahry, Probability density function approach for multidimensional turbulent flow calculations with application to in-cylinder flows in reciprocating engines, AIAA Journal 29 (2) (1991) 208-217.

[13] A. D. Leonard, F. Dash, Applications of a coupled Monte Carlo PDF finite volume CFD method for turbulent combustion, AIAA paper 94-2904 (1994).

[14] P. Eifler, W. Kollmann, PDF predictions of supersonic hydrogen flames, AIAA paper 93-0448 (1993).

[15] B. J. Delarue, S. B. Pope, Application of PDF methods to compressible turbulent flows, Physics of Fluids 9 (1997) 2704-2715.

[16] B. J. Delarue, S. B. Pope, Calculations of subsonic and supersonic turbulent reacting mixing layers using probability density function methods, Physics of Fluids 10 (1998) 487-498.

[17] A. T. Hsu, Y.-L. P., Tsai, M. S. Raju, Probability density function approach for compressible turbulent reacting flows, AIAA Journal 32 (2) (1994) 14071415. 
[18] H. Möbus, P. Gerlinger, D. Brüggemann, Monte Carlo PDF simulation of compressible turbulent diffusion flames using detailed chemical kinetics, AIAA paper 99-0198 (1999).

[19] F. A. Jaberi, P. J. Colucci, S. James, P. Givi, S. B. Pope, Filtered mass density function for large-eddy simulation of turbulent reacting flows, Journal of Fluid Mechanics 401 (1999) 85-121.

[20] H. Möbus, P. Gerlinger, D. Brüggemann, Comparison of Eularian and Lagrangian Monte Carlo PDF methods for turbulent diffusion flames, Combustion and Flame 124 (2001) 519-534.

[21] H. Möbus, P. Gerlinger, D. Brüggemann, Scalar and joint scalar-velocityfrequency Monte Carlo PDF simulation for supersonic combustion, Combustion and Flame 132 (2003) 3-24.

[22] A. Banaeizadeh, Z. Li, F. Jaberi, Compressible scalar filtered mass density function model for high-speed turbulent flows, AIAA Journal 49 (2011) 2130-2143.

[23] M. B. Nik, P. Givi, C. K. Madnia, S. B. Pope, EPVS-FMDF for LES of high-speed turbulent flows, AIAA paper 2012-117 (2012).

[24] P. R. Spalart, S. Deck, M. L. Squires, M. K. Strelets, A. Travin, A new version of detached-eddy simulations, resistant to ambiguous grid densities, Theoretical \& Computational Fluid Dynamics 20 (5) (2006) 3-24.

[25] X.-D. Liu, S. Osher, T. Chan, Weighted essentially non-oszillatory schemes, Journal of Computational Physics 115 (1994) 200-212.

[26] P. Gerlinger, D. Brüggemann, Multigrid convergence acceleration for turbulent supersonic flows, International Journal for Numerical Methods in Fluids 24 (1997) 1019-1035.

[27] P. Gerlinger, P. Stoll, D. Brüggemann, An implicit multigrid method for the simulation of chemically reacting flows, Journal of Computational Physics 146 (1998) 322-345. 
[28] P. Gerlinger, K. Nold, M. Aigner, Influence of reaction mechanisms, grid spacing, and inflow conditions on the numerical simulation of lifted supersonic flames, International Journal for Numerical Methods in Fluids 62 (2010) 1357-1380.

[29] P. Gerlinger, High-order multi-dimensional limiting for turbulent flows and combustion, Journal of Computational Physics 231 (2012) 137-170.

[30] T. J. Coakley, P. G. Huang, Turbulence modeling for high speed flows, AIAA paper 92-0436 (1992).

[31] A. Jameson, S. Yoon, Lower-upper implicit schemes with multiple grids for the Euler equations, AIAA Journal 25 (7) (1987) 929-935.

[32] J. S. Shuen, Upwind differencing and LU factorization for chemical nonequilibrium Navier Stokes equations, Journal of Computational Physics 99 (1992) 233-250.

[33] P. Gerlinger, H. Möbus, D. Brüggemann, An implicit multigrid method for turbulent combustion, Journal of Computational Physics 167 (2001) $247-276$.

[34] S.-H. Yoon, C. Kim, K.-H. Kim, Multi-dimensional limiting process for three-dimensional flow physics analyses, Journal of Computational Physics 227 (2008) 6001-6043.

[35] M.-S. Liou, A sequel to AUSM, part ii: $\mathrm{AUSM}^{+}$-up for all speeds, Journal of Computational Physics 214 (2006) 137-170.

[36] T. P. Coffee, J. M. Heimerl, Transport algorithms for premixed laminar steady state flames, Combustion and Flame 43 (1981) 273-289.

[37] K. K. Kuo, Principles of Combustion, John Wiley \& Sons New York, Chichester, Brisbane, Toronto, Singapore, 1986.

[38] P. Gerlinger, Numerische Verbrennungssimulation, Springer-Verlag Berlin Heidelberg, 2005, (in German). 
[39] P. H. Wang, S. B. Pope, Time-averaging strategies in the finitevolume/particle hybrid algorithm for the joint PDF equation of turbulent reactive flows, Combustion Theory and Modeling 12 (3) (2008) 529-544.

[40] R. C. Swanson, E. Turkel, J. A. White, An effective multigrid method for high speed flows, NASA, ICASE report No 91-56, contractor report 187602 (1991).

[41] E. Turkel, V. N. Vatsa, Effect of artificial viscosity on three dimensional flow simulations, AIAA paper 90-1444 (1990).

[42] M. Muradoglu, S. B. Pope, Local time-stepping algorithm for solving probability density function turbulence model equations, AIAA Journal 40 (9) (2002) 1755-1763.

[43] R. Kunz, B. Lakshminarayana, Stability of explicit Navier-Stokes procedures using $k-\epsilon$ and $k-\epsilon$ / algebraic Reynolds stress model, Journal of Computational Physics 103 (1992) 141-159.

[44] S. B. Pope, A probability approach to the modeling of turbulent reactive flows, Combustion and Flame 27 (1976) 299-312.

[45] A. T. Hsu, Y.-L. P., Tsai, M. S. Raju, A PDF approach for compressible turbulent reacting flows, AIAA paper 93-0087 (1993).

[46] A. Banaeizadeh, Z. Li, F. Jaberi, Large-eddy simulation of compressible turbulent reacting flows, AIAA paper 2010-202 (2010).

[47] D. C. Wilcox, Turbulence Modeling for CFD, DCW Industries, Inc., 2006.

[48] J. Villermaux, J. C. Devillon, Representation de la coalescence et de la redispersion des domaines de segragation dans un fluide par un modele d'interaction phenomenologique, in: Second International Symposium on Chemical Reaction Engineering, Elsevier, New York, 1972, pp. 1-13. 
[49] M. L. Shur, P. R. Spalart, M. K. Strelets, A. K. Travin, A hybrid RANSLES approach with delayed-DES and wall-modelled LES capabilities, International Journal of Heat and Fluid Flow 29 (2008) 1638-1649.

[50] A. Fiolitakis, P. Ess, P. Gerlinger, M. Aigner, Modeling of heat transfer and differential diffusion in transported PDF methods, Combustion and Flame 161 (2014) 2107-2119.

[51] R. O. Fox, Computational Models for Turbulent Reacting Flows, Cambridge University Press, 2003.

[52] S. Viswanathan, S. B. Pope, Turbulent dispersion from line sources in grid turbulence, Physics of Fluids 20 (2008) 101514-1-25.

[53] J. Pozorski, J.-P. Minier, Stochastic modelling of conjugate heat transfer in near-wall turbulence, International Journal of Heat and Fluid Flow 27 (2006) 867-877.

[54] D. G. Mabay, H. U. Mayer, G. W. Sawyer, Experimental and theoretical studies of the boundary layer on a flat plate at mach numbers from 2.5 to 4.5, RAE TR 74127 (1974).

[55] H. H. Fernholz, P. J. Finley, A critical compilation of compressible boundary layer data, AGARD-AG-223 (1977).

[56] J. McDaniels, D. Fletcher, R. H. Jr., S. Hallo, Staged transverse injection into Mach 2 flow behind a rearward facing step: A 3D compressible test case for hypersonic combustion code validation, AIAA paper 91-5071 (1991).

[57] R. L. Gaffney, J. A. White, S. S. Girimaji, J. P. Drummond, Modeling turbulenct/chemistry interactions using assumed PDF methods, AIAA paper 92-3638 (1992).

[58] P. Gerlinger, D. Brüggemann, Numerical investigation of hydrogen strut injections into supersonic airflows, Journal of Propulsion and Power 16 (2000) 22-28. 
[59] W. Marshall, S. Pal, R. Woodward, R. Santoro, Benchmark wall heat flux data for a $\mathrm{GO}_{2} / \mathrm{GH}_{2}$ single element combustor, AIAA paper 2005-3572 (2005).

[60] M. Lempke, R. Keller, P. Gerlinger, Influence of spatial discretization and unsteadyness on the simulation of rocket combustors, International Journal for Numerical Methods in Fluids 79 (9) (2015) 437-455. 